\title{
Interactions in nonconserving driven diffusive systems
}

\author{
Tripti Midha, ${ }^{1}$ Anatoly B. Kolomeisky, ${ }^{2}$ and Arvind Kumar Gupta ${ }^{1, *}$ \\ ${ }^{1}$ Department of Mathematics, Indian Institute of Technology Ropar, Rupnagar-140001, Punjab, India \\ ${ }^{2}$ Department of Chemistry and Center for Theoretical Biological Physics, Rice University, Houston, Texas 77005, USA
}

(Received 9 July 2018; published 9 October 2018)

\begin{abstract}
Motivated by biological transport phenomena and vehicular traffic flow we investigate the dynamics of interacting molecular motors or interacting vehicles that move along linear filaments (tracks) and can reversibly associate or dissociate from them. To analyze these processes, we introduced a model assimilating the interactions in a totally asymmetric simple exclusion process coupled with nonconserving Langmuir kinetics. The model is analyzed first using the continuum version of the simple mean-field approach that neglects the correlations between the particles. It is shown that even for weak interactions theoretical predictions deviate significantly from computer simulation results. To alleviate the problems, we developed a theoretical method that takes into account some correlations in the system. The effect of interactions on stationary phase diagrams, particle currents, and densities are explicitly evaluated. The analysis of two-point correlation function on the lattice indicates that the correlations are stronger at the locations of localized shocks. Our theoretical calculations are in excellent agreement with Monte Carlo computer simulations.
\end{abstract}

DOI: 10.1103/PhysRevE.98.042119

\section{INTRODUCTION}

A one-dimensional (1D) driven diffusive system (DDS) belongs to a special class of nonequilibrium systems that exhibit rich emergent properties [1-5]. A characteristic feature of these systems which distinguishes them from the equilibrium systems is the ever-present flow of particles in the steady state [6]. The DDS has become a popular research topic due to its wide applications, such as ribosomes motion along RNA [7,8], motor movement along molecular tracks [6], cars proceeding on highways $[9,10]$, vesicle locomotion [11], etc., occurring in physics, chemistry, and biology. The simplest driven model, the totally asymmetric simple exclusion process (TASEP), introduced originally in 1968 to model the kinetics of biopolymerization [12], has become a paradigmatic model in enlightening the nature of nonequilibrium steady states of DDS. In TASEP, a single species of particle hops unidirectionally with unit step size and a uniform rate along a 1D lattice. Here the particles interact only under the hard-core exclusion effect, which prohibits two or more particles from occupying the same lattice site at the same time. In the presence of open boundary conditions, the TASEP demonstrates several interesting phenomena, such as boundary-induced phase transitions, phase separation, and phase segregation $[2,13]$.

The simple TASEP when coupled with the equilibrium process, the Langmuir kinetics (LK), displays remarkable features, such as localized shock in the steady-state density profiles contributing to distinguishing high-low coexistence (shock) phase in the phase diagram [14,15]. The additional dynamics of the stochastic absorption and desorption processes (LK) in the bulk of TASEP was motivated by transport processes such as the beginning of a traffic jam on a motorway

\footnotetext{
*akgupta@iitrpr.ac.in
}

where incoming cars have to slow down very quickly over a short distance [16]. The finite processivity of molecular motors also leads to the switching between phases of directed and diffusive motion. During the latter, they are not attached to filament but move randomly through the cytoplasm under the constraints of other cytoskeletal elements [6]. The competition between the nonequilibrium process TASEP and the equilibrium process LK is possible when the attachment and detachment kinetic rates are rescaled such that they are inversely proportional to the system size. The simple TASEPLK model was theoretically analyzed using the continuum simple mean-field (SMF) approximation, and the obtained density and current profiles coincided with the Monte Carlo simulations $[14,15]$. Later, the formation of localized shocks in 1D DDS with LK was analyzed using the hydrodynamic approach [17]. It was argued there that the agreement of the simple mean-field (SMF) results with Monte Carlo simulations for the simple TASEP-LK system is due to the absence of correlations in the steady state of the system.

Several experiments provide evidence of the existence of mutual interactions among motor proteins, in particular kinesin-1. It is observed that in the presence of an obstacle, a molecular motor walking on a microtubule (molecular track) tends to stay attached for a longer time [18]. Nearest-neighbor repulsive interactions are also evident in traffic flow, where a car slows down if its next nearest-neighboring site is occupied [16]. Besides LK, the presence of mutual interactions among particles also has a major impact on the steady-state properties of DDS. The driven exclusion processes with nearestneighbor interactions and without LK have been extensively studied for a single $[1,16,19-29]$ as well as multilane TASEP systems [30,31], using the thermodynamically consistent [27-31] and phenomenological approaches [1,16,19-25]. The interactions in these systems are theoretically handled using the cluster mean-field and the modified cluster mean-field 
approaches. The major assumption in these theories is that the density and current are uniform throughout the lattice.

It is interesting to see how the system's dynamical properties behave when both the LK and the interactions are incorporated into the TASEP. In Ref. [17] it was predicted that the generic model of interacting driven diffusive systems, known as the Katz-Lebowitz-Spohn (KLS) model [1,19], in combination with LK can be explored using the hydrodynamic approach. Moreover, they observe that the KLS model with LK shows exotic features such as localized shocks and phase separation $[17,20]$. Recently, the effect of LK also has been investigated on the interacting TASEP systems, which view interactions in a thermodynamically consistent way [32]. The model was theoretically analyzed using the continuum SMF approach, and it was found that even in the presence of weak interactions, the density and the current profiles deviate from the simulation results [32]. However, neither the KLS model nor any other interacting system is explored with the effect of interactions on the LK process as well.

Experiments indicate a long-range cooperative binding of kinesin as well as the dependency of detachment rates on the biochemical state of the motors, which might be identified with the presence or absence of neighboring motors [33]. This sheds light on the importance of considering mutual interactions in the attachment-detachment processes as well. Further, it is also important to study the nonequilibrium steady-state properties of driven diffusive systems with LK, where the mutual interactions affect the dynamics of both the simple TASEP with open boundaries and the LK process. Recently the behavior of mutually interactive Langmuir kinetics coupled with the simple TASEP (without interactions) was analyzed using the continuum continuum SMF description and Monte Carlo simulations [34]. They observed that when mutual interactions affect the LK rates antisymmetrically, the continuum SMF results vary from the simulations. The reason behind the mismatching of the results is the ignorance of correlations by the SMF approximation. Moreover, the model had a major limitation that the mutual interactions are considered only in the LK process and not in the open TASEP. Since the interactions modify the dynamics of both the TASEP and the LK process, it becomes necessary and interesting to work on a model incorporating interactions for both the TASEP and the LK process. It also encourages development of a theoretical approach that can handle correlations in an open interactive TASEP-LK system.

To answer how the mutual interactions affect the combined dynamics of open TASEP and LK process, we present and study a model comprising interactive TASEP coupled with mutually interactive Langmuir kinetics.

In Sec. II we describe the model and obtain the master equations for the change in the average density profile. We analyze the system using the SMF approximation in Sec. III. We found that even in the presence of weak interactions, the results start deviating from the simulations, and for the large interactions the maximal particle current blows up. In Sec. IV we introduce an approach called the correlated cluster meanfield theory that takes into account the correlations between nearest-neighbor sites. In Sec. V we derive and discuss the impact of interactions on the phase diagrams and the density profiles. The current and the correlation profiles under the effect of interactions are analyzed in Sec. VI. We discuss the role of LK rates and finite system size on the density profiles and phase diagrams in Sec. VII. All theoretical results from the proposed theory are validated with Monte Carlo simulations. We conclude in Sec. VIII.

\section{MODEL DESCRIPTION AND MASTER EQUATIONS}

The model consists of a 1D open lattice with $N(N \gg$ 1) sites, which may represent a molecular track or a traffic highway. Correspondingly, the particles distributed over the lattice mimic the motor proteins such as kinesins, dyneins, and myosins on a molecular track or vehicles on a highway. The particles move unidirectionally in a random-sequential manner under the hard-core exclusion principle. Both ends of the lattice are coupled with two different reservoirs of constant density $\alpha$ and $1-\beta$, respectively, that drive the nonzero particle current into the system. We assign an occupation number $\tau_{i}$ to each lattice site $i(i=1,2, \ldots, N)$, which takes the value 1 when the site is occupied and 0 otherwise. First, we redefine the dynamics of the open simple TASEP-LK system in three subprocesses:

(i) Entrance of particles from the left reservoir to the site 1 with a rate $\alpha$ and their ejection from the site $N$ to the right reservoir with a rate $\beta$.

(ii) Hopping of a particle at site $i$ to site $i+1$, for $i=$ $1,2, \ldots, N-1$, with unit rate.

(iii) Stochastic attachment of particles from the surrounding environment to the bulk sites, $2,3, \ldots, N-1$, with a rate $\omega_{a}$ and detachment of the particles from the bulk sites to the surrounding environment with a rate $\omega_{d}$. This process is called Langmuir kinetics.

Note that in each of the above process, a particle can make a move only when the target site is empty, thus respecting the exclusion principle. To incorporate the effect of nearestneighbor particles' interactions in an open TASEP-LK system, we assume that the two nearest-neighboring particles, when bound to the filament, are associated with an energy $E$. We assign a rate $q$ for bond formation and a rate $r$ for bond breaking, where the rates $q$ and $r$ are related via

$$
\frac{q}{r}=e^{E}
$$

and $E$ is expressed in units of $k_{B} T$. The above relation follows from the visualization of the process of bond making and bond breaking as opposite reversible chemical reactions [27-29]. We also introduce a splitting parameter $\theta(0 \leqslant \theta \leqslant 1)$ that quantifies how the energy $E$ affects the hopping rates $q$ and $r$. The explicit values of rates $q$ and $r$ are then given as $q=e^{\theta E}$ and $r=e^{(\theta-1) E}$. We choose $\theta=0.5$, which splits interaction energy $E$ symmetrically on the rates $q$ and $r$, as well as incorporates the maximum effect of $E$ on both rates $q$ and $r$ simultaneously. It further makes the rates $q$ and $r$ inversely proportional to each other.

When the interactions are attractive $(E>0)$, the bond formation occurs faster, $q>1$, but the breaking of bonds occur slower, $r<1$, whereas in the case of repulsive interactions $(E<0)$, the system does not favor the bond making, implying $q<1$ and $r>1$. The case of repulsive interactions 


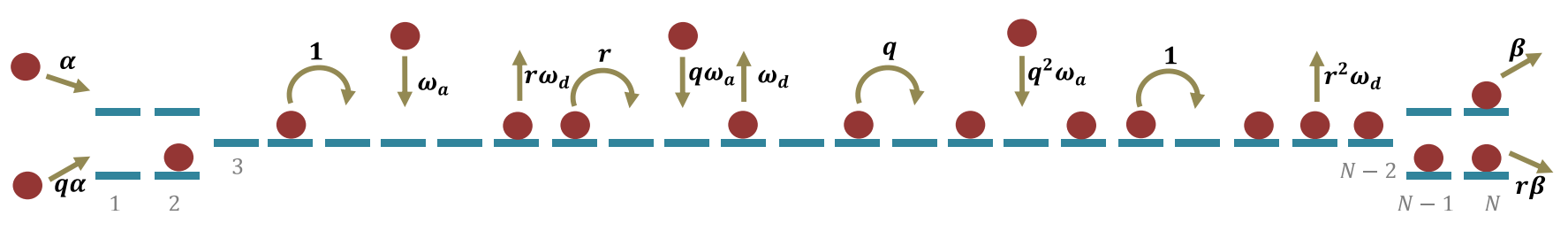

FIG. 1. Schematic view of the single lane TASEP-LK system under the effect of mutual interactions.

also reflects the realistic interactions found in vehicular traffic [16].

The consideration of mutual interactions in the open TASEP-LK system modifies the hopping rates of all its three subprocesses. When a particle hops, the number of bonds in the system may increase (when bonds form), decrease (when bonds break), or remain the same, i.e., the situation when either bonds break as well as form simultaneously or bonds neither form nor break concurrently. We define a parameter $s$ that represents the change in the number of bonds in the system in a one-time step. The parameter $s$ is positive or negative depending on the increment or decrement in the number of bonds, whereas the parameter $s$ takes the value 0 for no change in the total bonds in the system. For each increment (decrement) in the total quantity of bonds in a time step, the original rates of the TASEP-LK model get multiplied (divided) by a factor of $q(r)$. Figure 1 shows the modified hopping rates of the three subprocesses, under the interactions, which can be summarized as follows:

(1) In subprocess (i), the entrance and the exit rates depend on the occupancy state of sites 2 and $N-1$, respectively. A particle enters the empty site 1 with a rate $\alpha$ if site 2 is empty, otherwise with a rate $q \alpha$, since, in this case, the number of bonds increases by 1 . A particle at site $N$ leaves with a rate $\beta$ if site $N-1$ is empty, otherwise the rate of leaving is $r \beta$, which implies the system loses one bond [see Figs. 2(b) and 2(c)].

(2) In subprocess (ii), the hopping rate of a particle at sites $i=2,3, \ldots, N-2$, given that site $i+1$ is empty, depends

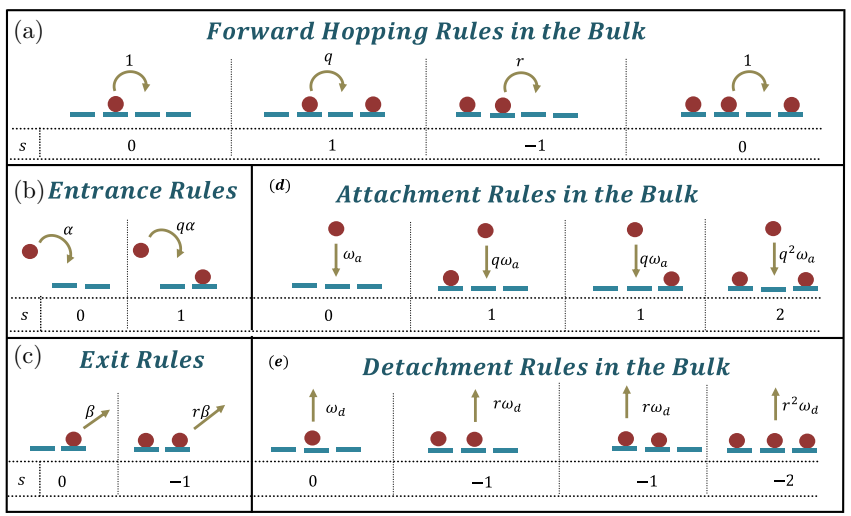

FIG. 2. The modified rules of interactive TASEP-LK system in terms of the increase and decrease in the number of bonds $(s)$ in the system. The parameter $s$ is positive or negative depending on the increment or decrement in the number of bonds. The parameter $s$ takes the value 0 for no change in the number of bonds in the system. For each increment (decrement), the original rates of TASEP-LK model get multiplied by a factor $q(r)$. upon the occupancy state of sites $i-1$ and $i+2$. For a particle at site $i=1(i=N-1)$, however, the rate depends only on the site $3(N-2)$. In the situation when the site $i-1$ is empty but site $i+2$ is occupied, the rate is $q$. Here the number of bonds in the system increases by one. But in the case when the site $i-1$ is occupied while the site $i+2$ is empty, the hopping rate is $r$, since, in this scenario, the number of bonds in the system decreases by one. In the other two cases, when both sites $i-1$ and $i+2$ are either empty simultaneously or occupied concurrently, the hopping rate is 1 because of no change in the number of bonds in the system [see Fig. 2(a)].

(3) In subprocess (iii), a particle's attachment and detachment rates for a bulk site $i$ depend on the occupancy state of its nearest-neighboring sites. The rate of attachment (detachment) is $\omega_{a}\left(\omega_{d}\right)$ when both the sites $i-1$ and $i+1$ are empty. In the case when either site $i-1$ or site $i+1$ is occupied, the attachment and detachment rate is, respectively, $q \omega_{a}$ and $r \omega_{d}$. In the last situation, when both the left and right neighboring sites of the $i$ th site are occupied, the attachment (detachment) rate becomes $q^{2} \omega_{a}\left(r^{2} \omega_{d}\right)$ [see Figs. 2(d) and 2(e)].

Thus, under the effect of interactions, the hopping rate of any subprocess of an original TASEP-LK system gets multiplied by a factor $q^{s}$, if the process leads to an increment of $s$ number of bonds, while the rate gets multiplied by $r^{-s}$, if the process causes a total of $s$ number of bond breakings in the system (see Fig. 2).

Clearly, in the absence of interactions $(E=0)$, the modified rates reduce to the original rates of three subprocesses of TASEP with a LK system.

\section{A. Master equations}

The temporal evolution of the average site occupation number $\left\langle\tau_{i}\right\rangle$ for any site $i(1 \leqslant i \leqslant N)$ consists of three terms: the inflow term $J_{i-1, i}$ from site $i-1$ to site $i$, the drain term $J_{i, i+1}$ from site $i$ to site $(i+1)$, and the respective, stochastic attachment $S_{i, A}$ and detachment $S_{i, D}$ term, to and from the site $i$. The master equation for the probability of the occupied $i$ th site, $P\left(\tau_{i}=1\right)$, for $1 \leqslant i \leqslant N$, is thus read as

$$
\frac{d\left\langle\tau_{i}\right\rangle}{d t}=J_{i-1, i}-J_{i, i+1}+S_{i, A}-S_{i, D} .
$$

According to the system's dynamics, the particle current from site $i$ to site $i+1(2 \leqslant i \leqslant N-2)$, depending on the occupancy of nearest- and next-nearest-neighboring sites, is given as

$$
\begin{aligned}
J_{i, i+1}= & \left\langle\tilde{\tau}_{i-1} \tau_{i} \tilde{\tau}_{i+1} \tilde{\tau}_{i+2}\right\rangle+q\left\langle\tilde{\tau}_{i-1} \tau_{i} \tilde{\tau}_{i+1} \tau_{i+2}\right\rangle \\
& +r\left\langle\tau_{i-1} \tau_{i} \tilde{\tau}_{i+1} \tilde{\tau}_{i+2}\right\rangle+\left\langle\tau_{i-1} \tau_{i} \tilde{\tau}_{i-1} \tau_{i+2}\right\rangle
\end{aligned}
$$


where $\tilde{\tau}_{i}=1-\tau_{i}$. The particle current for the entrance at site $i=1$ is given as

$$
J_{\mathrm{entr}}=\alpha\left\langle\tilde{\tau}_{1} \tilde{\tau}_{2}\right\rangle+q \alpha\left\langle\tilde{\tau}_{1} \tau_{2}\right\rangle
$$

Further, the particle current from site 1 to the empty site 2 depending on the occupancy state of site 3 is given as

$$
J_{1,2}=\left\langle\tau_{1} \tilde{\tau_{2}} \tilde{\tau}_{3}\right\rangle+q\left\langle\tau_{1} \tilde{\tau}_{2} \tau_{3}\right\rangle
$$

Similarly, a particle's flow to the last site depending on the occupancy of the last three sites is written as

$$
J_{N-1, N}=\left\langle\tilde{\tau}_{N-2} \tau_{N-1} \tilde{\tau}_{N}\right\rangle+r\left\langle\tau_{N-2} \tau_{N-1} \tilde{\tau}_{N}\right\rangle,
$$

and the exit current from the lattice depending on the last two sites of the lattice is read as

$$
J_{\text {exit }}=\beta\left\langle\tilde{\tau}_{N-1} \tau_{N}\right\rangle+r \beta\left\langle\tau_{N-1} \tau_{N}\right\rangle .
$$

Similarly, the attachment and detachment terms for any bulk site $i(2 \leqslant i \leqslant N-1)$, which depend on the occupancy states of the left and the right nearest-neighboring sites, are stated as

$$
\begin{aligned}
S_{i, A}= & \omega_{a}\left\langle\tilde{\tau}_{i-1} \tilde{\tau}_{i} \tilde{\tau}_{i+1}\right\rangle+q^{2} \omega_{a}\left\langle\tau_{i-1} \tilde{\tau}_{i} \tau_{i+1}\right\rangle \\
& +q \omega_{a}\left(\left\langle\tilde{\tau}_{i-1} \tilde{\tau}_{i} \tau_{i+1}\right\rangle+\left\langle\tau_{i-1} \tilde{\tau}_{i} \tilde{\tau}_{i+1}\right\rangle\right)
\end{aligned}
$$

and

$$
\begin{aligned}
S_{i, D}= & \omega_{d}\left\langle\tilde{\tau}_{i-1} \tau_{i} \tilde{\tau}_{i+1}\right\rangle+r^{2} \omega_{d}\left\langle\tau_{i-1} \tau_{i} \tau_{i+1}\right\rangle \\
& +r \omega_{d}\left(\left\langle\tau_{i-1} \tau_{i} \tilde{\tau}_{i+1}\right\rangle+\left\langle\tilde{\tau}_{i-1} \tau_{i} \tau_{i+1}\right\rangle\right) .
\end{aligned}
$$

For simplicity, we have not considered the attachment and detachment at the first and last sites. Equations (3)-(8) are further simplified in Appendix A. By substituting Eqs. (A1)(A7) in Eq. (2), we can obtain the master equations for the single-site probability $\rho_{i}$ regarding one-, two-, three-, and four-site joint probabilities.

\section{SIMPLE MEAN-FIELD APPROXIMATION AND CONTINUUM LIMIT}

The computation of time evolution of the probability of average occupancy of the $i$ th site, $\left\langle\tau_{i}(t)\right\rangle$, and the particle current requires the determination of one-, two-, three-, and four-point correlators. We first consider the SMF approximation, which ignores the particles' spatial correlations and replaces the higher order correlators with the product of individual average site occupations:,

$$
\begin{aligned}
\left\langle\tau_{i} \tau_{j}\right\rangle & \approx\left\langle\tau_{i}\right\rangle\left\langle\tau_{j}\right\rangle, \\
\left\langle\tau_{i} \tau_{j} \tau_{k}\right\rangle & \approx\left\langle\tau_{i}\right\rangle\left\langle\tau_{j}\right\rangle\left\langle\tau_{k}\right\rangle, \text { and } \\
\left\langle\tau_{i} \tau_{j} \tau_{k} \tau_{l}\right\rangle & \approx\left\langle\tau_{i}\right\rangle\left\langle\tau_{j}\right\rangle\left\langle\tau_{k}\right\rangle\left\langle\tau_{l}\right\rangle .
\end{aligned}
$$

Defining $\rho_{i}=\left\langle\tau_{i}\right\rangle$ and utilizing the SMF approximation, the expressions of the particle current reduce to

$$
\begin{gathered}
J_{i, i+1}=\rho_{i}\left(1-\rho_{i+1}\right)\left[1+(q-1) \rho_{i+2}\left(1-\rho_{i-1}\right)\right. \\
\left.+(r-1) \rho_{i-1}\left(1-\rho_{i+2}\right)\right], \\
J_{1,2}=\rho_{1}\left(1-\rho_{2}\right)\left[1+(q-1) \rho_{3}\right], \\
J_{N-1, N}=\rho_{N-1}\left(1-\rho_{N}\right)\left[1+(r-1) \rho_{N-2}\right], \\
J_{\text {entr }}=\alpha\left(1-\rho_{1}\right)\left[1+(q-1) \rho_{2}\right], \quad \text { and } \\
J_{\text {exit }}=\beta \rho_{N}\left[1+(r-1) \rho_{N-1}\right] .
\end{gathered}
$$

In the absence of interactions, $q=r=1$, the above expressions of the particle current reduce to the corresponding terms of the simple TASEP-LK system [15].

Similarly, under the SMF approximation, the attachment and detachment terms are simplified to

$$
\begin{gathered}
S_{i, A}=\omega_{a}\left(1-\rho_{i}\right)\left[1+(q-1) \rho_{i-1}\right]\left[1+(q-1) \rho_{i+1}\right], \\
S_{i, D}=\omega_{d} \rho_{i}\left[1+(r-1) \rho_{i-1}\right]\left[1+(r-1) \rho_{i+1}\right] .
\end{gathered}
$$

When there is no interaction $(E=0)$, the above equations, respectively, reduce to $S_{i, A}=\omega_{a}\left(1-\rho_{i}\right)$ and $S_{i, D}=\omega_{d} \rho_{i}$ [15].

Utilizing Eqs. (11)-(17), the master equation [Eq. (2)] of the average density in the steady state becomes

$$
\begin{aligned}
& \rho_{i-1}\left(1-\rho_{i}\right)\left[1+(q-1) \rho_{i+1}\left(1-\rho_{i-2}\right)+(r-1) \rho_{i-2}\left(1-\rho_{i+1}\right)\right]-\rho_{i}\left(1-\rho_{i+1}\right)\left[1+(q-1) \rho_{i+2}\left(1-\rho_{i-1}\right)\right. \\
& \left.\quad+(r-1) \rho_{i-1}\left(1-\rho_{i+2}\right)\right]+\omega_{a}\left(1-\rho_{i}\right)\left[1+(q-1) \rho_{i-1}\right]\left[1+(q-1) \rho_{i+1}\right]-\omega_{d} \rho_{i}\left[1+(r-1) \rho_{i-1}\right]\left[1+(r-1) \rho_{i+1}\right]=0
\end{aligned}
$$

for the bulk sites. In the case of the first and the last sites, we get

$$
\alpha\left(1-\rho_{1}\right)\left[1+(q-1) \rho_{2}\right]-\rho_{1}\left(1-\rho_{2}\right)\left[1+(q-1) \rho_{3}\right]=0
$$

and

$$
\rho_{N-1}\left(1-\rho_{N}\right)\left[1+(r-1) \rho_{N-2}\right]-\beta \rho_{N}\left[1+(r-1) \rho_{N-1}\right]=0,
$$

respectively. To obtain a continuum description of Eqs. (18)(20) in the hydrodynamic limit $N \rightarrow \infty$, we coarse grain the discrete lattice, assuming the lattice length to be equal to 1 , with lattice constant $\epsilon=1 / N$, and rescale the time variable as $t^{\prime}=t / N$. For large $N$, we have the quasicontinuous rescaled position variable $x=i \epsilon, 0 \leqslant x \leqslant 1$. To ensure the competing 
interplay between the conserving and nonconserving dynamics, we rescale the attachment and detachment rates in such a way that the kinetic rates decrease simultaneously with an increase in the system size:

$$
\Omega_{a}=\omega_{a} N, \quad \Omega_{d}=\omega_{d} N .
$$

Performing a Taylor expansion of the average density $\rho(x) \equiv$ $\rho_{i}$ in powers of $\epsilon$,

$$
\rho(x \pm n \epsilon)=\rho(x) \pm n \epsilon \partial_{x} \rho(x)+\frac{1}{2}(n \epsilon)^{2} \partial_{x}^{2} \rho(x)+O\left(\epsilon^{3}\right),
$$

in Eq. (18), one can easily obtain the equation of average density as a function of $t^{\prime}$ and quasicontinuous space variable $x$, described as

$$
\begin{aligned}
\frac{\partial \rho}{\partial t^{\prime}}= & \frac{\epsilon}{2} \frac{\partial^{2} \rho}{\partial x^{2}}-\frac{\partial J}{\partial \rho} \frac{\partial \rho}{\partial x}+\Omega_{a}(1-\rho)[1+(q-1) \rho]^{2} \\
& -\Omega_{d} \rho[1+(r-1) \rho]^{2},
\end{aligned}
$$

where $\quad J=\rho(1-\rho)[1+(q-1) \rho(1-\rho)+(r-1) \rho(1-$ $\rho)$ ] defines the bulk particle current. Similarly, Eqs. (19) and (20) are, respectively, translated to the following boundary conditions:

$$
\rho(0)=\alpha, \quad \rho(1)=1-\beta .
$$

In the steady state and inviscid limit of $\epsilon \rightarrow 0$, the secondorder nonlinear equation [Eq. (23)] reduces to the following first-order nonlinear differential equation:

$$
\frac{\partial \rho}{\partial x}=\frac{\Omega_{a}(1-\rho)[1+(q-1) \rho]^{2}-\Omega_{d} \rho[1+(r-1) \rho]^{2}}{(1-2 \rho)^{3}+2(q+r) \rho(1-\rho)(1-2 \rho)},
$$

with the boundary conditions given by Eq. (24). This boundary value problem is over-determined due to the presence of two boundary conditions. However, the solutions of the equation can be obtained by satisfying only one of the boundary conditions at a time. The solution obeying the left boundary condition is denoted as $\rho_{\alpha}$, while the one satisfying the right boundary condition is represented as $\rho_{\beta}$. To obtain the constant Langmuir isotherm solution $\left(\rho_{l}\right)$, we equate Eq. (25) to 0 , which yields the following third-order equation:

$$
\begin{gathered}
\rho^{3}\left[K(q-1)^{2}+(r-1)^{2}\right]-\rho^{2}[K(q-1)(q-3) \\
-2(r-1)]-\rho[K(2 q-3)-1]-K=0,
\end{gathered}
$$

where $K=\frac{\Omega_{a}}{\Omega_{d}}$ represents the binding constant. According to Cardano's formula, we find that the above third-order equation always has only one real root belonging to $(0,1)$ for any particular value of $K$ and $E$. We denote the real root as the Langmuir isotherm $\left(\rho_{l}\right)$, which is independent of both the left and the right boundaries but depends on the interaction energy $E$ and the binding constant $K$. In the absence of interactions, the above equation gives the Langmuir isotherm density, $\rho_{l}=$ $\frac{K}{K+1}$, for the simple TASEP-LK system [14]. For $0<\epsilon \ll$ 1 , the full density profile in the range $0 \leqslant x \leqslant 1$, i.e., the complete steady-state solution of Eq. (23), is constructed from the possible combination of the three solutions $[15,34]$

$$
\rho(x)= \begin{cases}\rho_{\alpha} & \text { for } \quad 0 \leqslant x \leqslant x_{\alpha} \\ \rho_{l} & \text { for } \quad x_{\alpha} \leqslant x \leqslant x_{\beta} \\ \rho_{\beta} & \text { for } \quad x_{\beta} \leqslant x \leqslant 1 .\end{cases}
$$

The corresponding position $x_{\alpha}\left(x_{\beta}\right)$ separating $\rho_{\alpha}\left(\rho_{\beta}\right)$ from $\rho_{l}$ is obtained by matching the corresponding currents $J(\rho)$ :

$J(\rho)=[\rho(1-\rho)]\left[(1-\rho)^{2}+\rho^{2}+(q+r) \rho(1-\rho)\right]$.

For $x_{\alpha}$, the current, $J_{\alpha}\left(x_{\alpha}\right)$, corresponding to the left solution $\rho_{\alpha}$ is matched with the Langmuir current $J_{l}=J\left(\rho_{l}\right)$, while the position $x_{\beta}$ is obtained by equating the right solution current $J_{\beta}\left(x_{\beta}\right)$ with the Langmuir current $J_{l}$.

In general, it happens that the left solution $\left(\rho_{\alpha}\right)$ does not continuously match the right solution $\left(\rho_{\beta}\right)$. For the limit $\epsilon \rightarrow$ $0^{+}$, the crossover interval $\left(x_{\alpha}, x_{\beta}\right)$ decreases in width and leads to a discontinuity of the average density profile at some position $x_{w}$. In this case, the density profile is given as

$$
\rho(x)=\left\{\begin{array}{lll}
\rho_{\alpha} & \text { for } & 0 \leqslant x \leqslant x_{w} \\
\rho_{\beta} & \text { for } & x_{w} \leqslant x \leqslant 1 .
\end{array}\right.
$$

Here the Langmuir density $\rho_{l}$ is not a part of the density profile, and $x_{w}$ is obtained by equating the current corresponding to both the solutions at $x_{w}: J_{\alpha}\left(x_{w}\right)=J_{\beta}\left(x_{w}\right)$.

\section{A. Shortcomings of the SMF approximation}

We now highlight the major shortcomings of the SMF approximation and its continuum limit in the analyses of the steady-state properties of the interactive TASEP-LK system. First, we observe that the phase diagrams as well as the density and the current profiles in every phase of a phase diagram obtained from the continuum SMF approximation [see Figs. 3(b), 3(c), 5, 6, 8, and 9] deviate significantly from the

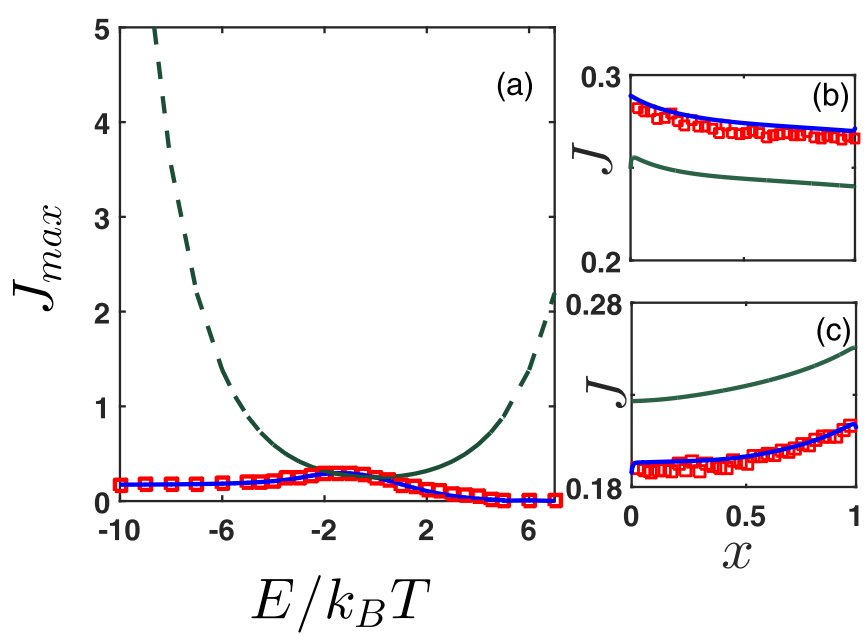

FIG. 3. (Left) (a) Maximal particle current $J_{M C}$ as a function of interaction energy $E$ in the maximal current phase. The maximal particle current exists in the MC phase of every phase diagram for the interaction energy $E$ and its corresponding critical binding constant $K^{*}$ computed in Sec. V B. Moreover, the maximal current occurs for weak repulsion, which matches with the strength of the interactive TASEP system without LK. (Right) Current profile in the Meissner phase for $\Omega_{d}=0.3, K=1$ and interaction strength (b) $E=-0.6 k_{B} T$; (c) $E=0.6 k_{B} T$. For simulations, $\alpha=1, \beta=1$ is utilized. Symbols are the results of Monte Carlo simulations with a lattice site of 1000 sites, averaged over $5 \times 10^{9}$ simulations. Solid and dashed lines, respectively, represent the correlated cluster meanfield and SMF results. 

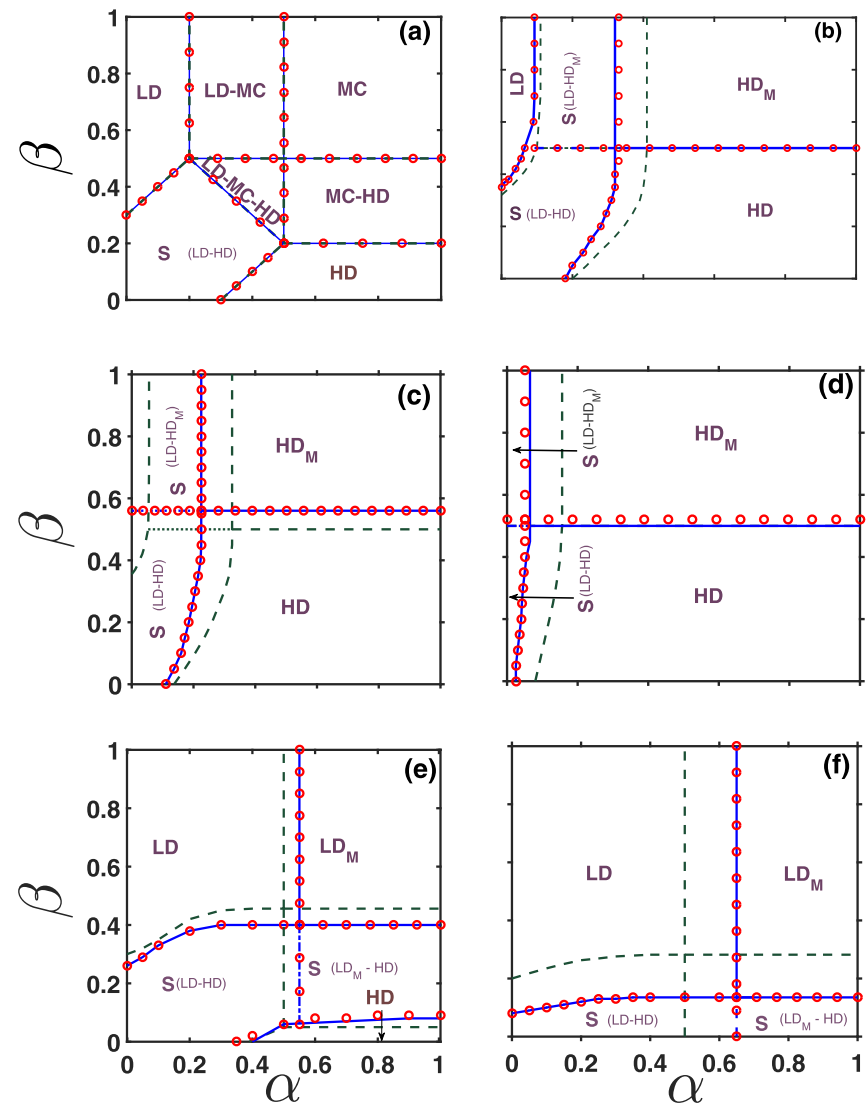

FIG. 4. Phase diagrams for interactive TASEP with mutually interactive Langmuir kinetics for $\Omega=0.3, K=1$ and (a) $E=$ $0.0 k_{B} T$; (b) $E=0.3 k_{B} T$ (c) $E=0.6 k_{B} T$; (d) $E=1.6 k_{B} T$; (e) $E=-0.2 k_{B} T$; (f) $E=-1.6 k_{B} T$. Solid and dashed lines, respectively, indicate the correlated cluster mean-field and SMF results. Symbols represent the simulation results. For $E=0.0 k_{B} T$, the correlated cluster mean-field results match exactly with the SMF and simulation results [15].

Monte Carlo simulations, for any value of binding constant, $K$, and interaction strength, $E$. Moreover, the deviations in the results increase with the rise in the strength $E$, leading to not only qualitative changes but also quantitative changes in the phase diagram. For instance, in Fig. 4(c), the continuum SMF theory predicts a low-density phase near the upper left quadrant, whereas the phase does not exist according to the simulations.

Second, the particle maximal current phase exists for every interaction energy $E$ only at a critical binding constant $K^{*}(E)$ (to be discussed in Sec. V B). In the maximal current phase, the SMF theory approximates the particle current as $J_{\max }=$ $1 / 8+((q+r) / 16)$, which is obtained by substituting the critical density $\rho_{\max }=1 / 2$ in the bulk current $J$. Figure 3 (a) shows that the maximal particle current, $J_{\max }$, obtained from the SMF theory, increases without bound for $|E| \gg 1$, which is neither realistic nor agrees with simulations. In the presence of large attractive interactions, the particles group together to form large clusters, which hinder their movement, while for infinite repulsions, they behave as noninteracting dimers with a finite nonzero particle current [8]. Note that the unbounded maximal current for the conserved system continues to prevail in the considered nonconserved system [17]. Thus, the above drawbacks point out the failure of the SMF approximation and its continuum limit in computing the steady-state properties of the TASEP-LK system under weak as well as strong interactions. To deal with the nearest-neighbor interactions in the TASEP-LK system, we define an approach in the next section that can handle correlations and can overcome all the pitfalls of the SMF approximation.

\section{CORRELATED CLUSTER MEAN-FIELD THEORY}

The theoretical examination of the TASEP-LK system in the presence of mutual interactions requires an efficient analytical approach that takes into account the correlations to some extent and works well for nonconserving systems. The existing theoretical studies such as cluster mean-field theory [27,29], modified cluster mean-field theory [28], timedensity functional approach [23], etc., are efficient only for the conserved systems. These theories assume that any observable is uniform and independent of the position on the lattice. Since the TASEP along with the LK process produces nonuniform, nonlinear density profiles $[34,35]$ as well as localized shocks in the density profiles, these theories cannot be directly utilized for the interacting TASEP-LK system. It indicates that the dealing of the interactive TASEP-LK system requires an efficient, generalized approach, which we call a correlated cluster mean-field (CCMF) approach, that successfully handles the nearest-neighbor correlations in the system.

The $n$-CCMF approach, in general, exactly considers the correlations between $n$ consecutive sites in the state (say) $\left\{\sigma_{i}, \sigma_{i+1}, \ldots, \sigma_{i+n-1}\right\}$, where $\sigma_{i}$ represents the particle or hole occupation number of the $i$ th site. We consider the case of $n=2$, in which the CCMF approach exactly considers the correlations between two nearest-neighboring sites. Taking into account the particle-hole symmetry, a pair of nearestneighboring sites can exist in any of the four possible states: $\{1,1\},\{1,0\},\{0,1\}$, and $\{0,0\}$. For simplification, we denote the two-point nearest-neighbor correlator $\left\langle\tau_{i} \tau_{i+1}\right\rangle$ or the probability when both sites $i$ and $i+1$ are occupied, i.e., $P\left(\tau_{i}=\right.$ $\left.1, \tau_{i+1}=1\right)$, by $f_{i}$. Then, using the Kolmogorov consistency conditions, the remaining two-site cluster probabilities can be obtained as follows:

$$
\begin{aligned}
\left\langle\tau_{i}\left(1-\tau_{i+1}\right)\right\rangle & =\rho_{i}-f_{i}, \\
\left\langle\left(1-\tau_{i}\right) \tau_{i+1}\right\rangle & =\rho_{i+1}-f_{i}, \quad \text { and } \\
\left\langle\left(1-\tau_{i}\right)\left(1-\tau_{i+1}\right)\right\rangle & =1-\rho_{i}-\rho_{i+1}+f_{i} .
\end{aligned}
$$

The correlator $\left\langle\sigma_{i} \sigma_{i+1} \sigma_{i+2}\right\rangle$ between the three consecutive sites $i, i+1$, and $i+2$ in their respective occupation states $\sigma_{i}, \sigma_{i+1}$, and $\sigma_{i+2}$, is approximated as

$$
\left\langle\sigma_{i} \sigma_{i+1} \sigma_{i+2}\right\rangle \propto\left\langle\sigma_{i} \sigma_{i+1}\right\rangle\left\langle\sigma_{i+1} \sigma_{i+2}\right\rangle,
$$

which is further factorized in terms of a one- and two-point correlator as [36-38]

$$
\left\langle\sigma_{i} \sigma_{i+1} \sigma_{i+2}\right\rangle=\frac{\left\langle\sigma_{i} \sigma_{i+1}\right\rangle\left\langle\sigma_{i+1} \sigma_{i+2}\right\rangle}{\left\langle\sigma_{i+1}\right\rangle},
$$

provided the term in the denominator is nonzero. The above equation can be understood as follows. For a cluster of size 3, Eq. (32) exactly considers the correlations between the 
clusters made up of two nearest-neighboring sites, i.e., for a cluster of sites $i, i+1$, and a cluster of sites $i+1, i+2$. Since it involves the double consideration of the probability of the occupancy of site $i+1$, the approximation must be corrected with the division of the probability of the occupancy of site $i+1$, which yields Eq. (32) [36-38]. In general, the correlations between $n$ consecutive sites are factorized in terms of one- and two-point correlators as

$$
\left\langle\sigma_{1} \sigma_{2} \cdots \sigma_{n}\right\rangle=\frac{\left\langle\sigma_{1} \sigma_{2}\right\rangle\left\langle\sigma_{2} \sigma_{3}\right\rangle \cdots\left\langle\sigma_{n-1} \sigma_{n}\right\rangle}{\left\langle\sigma_{2}\right\rangle\left\langle\sigma_{3}\right\rangle \cdots\left\langle\sigma_{n-1}\right\rangle},
$$

provided the terms in the denominator are nonzero [36-38]. Similarly, here, for a cluster of size $n$, only the probabilities between the cluster made up of two nearest-neighboring sites are considered exactly, i.e., between sites $j$ and $j+1$, where $1 \leqslant j \leqslant n-1$. The probabilities of the bulk sites $\{2,3, \ldots, n-1\}$, which are included twice in the numerator, are divided to correct the approximation. Thus, from the application of Eq. (33), any higher order correlator can be written as a function of average density $\rho_{i}$ and the average two-point correlator $f_{i}$, corresponding to the site $i$.

Basically the CCMF approach works in two steps. First, the higher order correlators, i.e., three- and more point nearestneighbor correlators, appearing in the time evolution of the average density $\rho$, and two-point correlator $f$ are reduced regarding one- and two-point correlators by using the relations (30)-(33). Second, the density, $\rho$, and the two-point correlator, $f$, are numerically obtained from a pair of coupled secondorder nonlinear ordinary differential equations in the two unknowns $\rho$ and $f$ in respect to the boundary conditions. The density and the two-point correlator function can then be utilized to obtain the system's other properties.

We now move to step 1 and first write the master equation governing the time evolution of average density $\rho$. However, the master equation for $\rho$ remains intact as Eq. (2), but the gain terms $\left(J_{i-1, i}\right.$ and $\left.S_{i, A}\right)$ and the loss terms $\left(J_{i, i+1}\right.$ and $\left.S_{i, D}\right)$, given by Eqs. (3)-(9), reduce to functions of only one- and two-point correlators, by using the correlated cluster meanfield approximation [Eqs. (30)-(33)].

The simplified form of the bulk particle current $J_{i, i+1}$, given by Eq. (3), under the CCMF approximation is written as

$$
\begin{aligned}
J_{i, i+1}= & \left(\rho_{i}-f_{i}\right)+(r-1) f_{i-1}\left(1-\frac{f_{i}}{\rho_{i}}\right) \\
& +\left[\frac{\left(\rho_{i}-f_{i}\right)\left(\rho_{i+2}-f_{i+1}\right)}{1-\rho_{i+1}}\right] \\
& \times\left[(q-1)+\frac{(2-q-r) f_{i-1}}{\rho_{i}}\right] .
\end{aligned}
$$

In the absence of interactions, $q=r=1$, the above equation reduces to $J_{i, i+1}=\rho_{i}-f_{i}$, which matches the case of TASEP without interactions [29,39]. We claim that Eq. (34) overcomes the major drawback of the SMF approximation in producing the unbounded current for large interactions. To prove our claim, we rewrite Eq. (34), assuming the current to be uniform for sites far from the boundaries, as follows:

$$
\begin{aligned}
J= & (\rho-f)+(r-1) f\left(1-\frac{f}{\rho}\right) \\
& +\left[\frac{(\rho-f)^{2}}{1-\rho}\right]\left[(q-1)+\frac{(2-q-r) f}{\rho}\right] .
\end{aligned}
$$

The uniform two-site cluster probability $f=P(1,1)$ can be determined by solving its steady-state master equation, which yields the relation $q(\rho-f)^{2}=r f(1-2 \rho+f)$, and further gives

$$
f= \begin{cases}\frac{1}{2}\left[2 \rho+\frac{r-\sqrt{r[r+4(q-r) \rho(1-\rho)]}]}{(q-r)}\right] & \text { if } q, r \neq 1 \\ \rho^{2} & \text { if } q=r=1 .\end{cases}
$$

Utilizing Eq. (36) in Eq. (35), we obtain the particle current as a function of interaction energy $E$ and density $\rho$. The plot of the maximal values of particle current corresponding to various values of interaction energy $E$ agrees very well with the Monte Carlo simulations [see Fig. 3(a)].

For the special case of $E \rightarrow-\infty$, where particles behave as noninteracting dimers, we obtain $f \rightarrow 0$ and $J \rightarrow \frac{\rho(1-2 \rho)}{(1-\rho)}$ [41]. For the case when $E \rightarrow \infty$, we get $f \rightarrow \rho$ and $J \rightarrow 0$, which is well justified with the physical explanation given in Sec. III A. Thus, the proposed CCMF approach overcomes the drawback of an unbounded particle current with respect to the interaction energy $E$.

We now write the simplified expressions of the inflow and outflow current for sites 1 and $N$, respectively, using Eqs. (30)-(33) in Eqs. (4)-(7) as follows:

$$
\begin{gathered}
J_{\mathrm{entr}}=\alpha\left(1-\rho_{1}\right)+\alpha(q-1)\left(\rho_{2}-f_{1}\right), \\
J_{1,2}=\left(\rho_{1}-f_{1}\right)\left[1+(q-1) \frac{\rho_{3}-f_{2}}{1-\rho_{2}}\right] \\
J_{N-1, N}=\left(\rho_{N-1}-f_{N-1}\right)+(1-r) f_{N-2}\left(\frac{f_{N-1}}{\rho_{N-1}}-1\right),
\end{gathered}
$$

$$
J_{\text {exit }}=\beta\left(\rho_{N}+(r-1) f_{N-1}\right) .
$$

For $E \rightarrow 0$, we obtain $J_{\text {entr }} \rightarrow \alpha\left(1-\rho_{1}\right)$ and $J_{\text {exit }} \rightarrow \beta \rho_{N}$ [15]. When $E \rightarrow-\infty$, we acquire $J_{\text {entr }} \rightarrow \alpha\left(1-\rho_{1}-\rho_{2}\right)$ and $J_{\text {exit }} \rightarrow \beta \rho_{N}$ [40]. The case of infinite attractions produces $J_{\text {entr }}, J_{\text {exit }} \rightarrow 0$.

TABLE I. Gain term, $F_{\text {in }}$, in the master equation for the two-point correlator function $f_{i}$.

\begin{tabular}{lcc}
\hline \hline Site & $F_{\text {in }}$ & $F_{\text {in }}$ (CCMF approximation) \\
\hline$i=1$ & $q \alpha\left\langle\left(1-\tau_{1}\right) \tau_{2}\right\rangle$ & $q \alpha\left(\rho_{2}-f_{1}\right)$ \\
$i=2$ & $q\left\langle\tau_{i-1}\left(1-\tau_{i}\right) \tau_{i+1}\right\rangle$ & $q\left[\frac{\left(\rho_{i-1}-f_{i-1}\right)\left(\rho_{i+1}-f_{i}\right)}{1-\rho_{i}}\right]$ \\
$3 \leqslant i \leqslant N-1$ & $q\left\langle\tau_{i-1}\left(1-\tau_{i}\right) \tau_{i+1}\right\rangle+(1-q)\left\langle\tau_{i-2} \tau_{i-1}\left(1-\tau_{i}\right) \tau_{i+1}\right\rangle$ & {$\left[q+(1-q) \frac{f_{i-2}}{\rho_{i-2}}\right]\left[\frac{\left.\rho_{i-1}-f_{i-1}\right)\left(\rho_{i+1}-f_{i}\right)}{1-\rho_{i}}\right]$} \\
\hline \hline
\end{tabular}


In a similar way, the source and sink terms [Eqs. (8) and (9)] are simplified using the CCMF theory [Eqs. (30)-(32)], as follows:

$$
\begin{aligned}
S_{i, A}= & \omega_{a}\left(\left(1-\rho_{i}\right)+(q-1)\left\{\left(\rho_{i+1}-f_{i}\right)+\left(\rho_{i-1}-f_{i-1}\right)\right.\right. \\
& \left.\left.\times\left[1+\frac{(q-1)\left(\rho_{i+1}-f_{i}\right)}{1-\rho_{i}}\right]\right\}\right), \\
S_{i, D}= & \omega_{d}\left(\rho_{i}+(r-1)\left\{f_{i}+f_{i-1}\left[1+\frac{(r-1) f_{i}}{\rho_{i}}\right]\right\}\right),
\end{aligned}
$$

where $1<i<N$. Clearly, $E \rightarrow 0$, yields $S_{i, A} \rightarrow \omega_{a}\left(1-\rho_{i}\right)$ and $S_{i, D} \rightarrow \omega_{d} \rho_{i}$ [15]. When $E \rightarrow-\infty$, we get $f_{i} \rightarrow 0$ and, thus, $S_{i, A} \rightarrow \frac{\Omega_{a}\left(1-\rho_{i-1}-\rho_{i}\right)\left(1-\rho_{i}-\rho_{i+1}\right)}{1-\rho_{i}}, S_{i, D} \rightarrow \omega_{d} \rho_{i}$ [41]. Since there is no attachment and detachment at the first and last sites, we have $S_{i, A}=S_{i, D}=0$, for $i=1$ and $N$.

Substituting Eqs. (34) and (37)-(42) in Eq. (2), one can obtain the temporal evolution of density $\left(\rho_{i}\right)$ at any site $i$ as a nonlinear combination of correlation function $f$ and density function $\rho$ corresponding to the sites $i-1, i, i+1$, and $i+2$.We now write the master equation governing the time evolution of the two-point correlator $f_{i}=\left\langle\tau_{i} \tau_{i+1}\right\rangle$, depending on the neighboring sites, as follows:

$$
\frac{d\left\langle\tau_{i} \tau_{i+1}\right\rangle}{d t}=F_{i, \text { in }}-F_{i, \text { out }}+F_{i, A}-F_{i, D},
$$

where $F_{i, \text { in }}$ and $F_{i, \text { out }}$, respectively, denote the gain and the loss terms to the joint probability of the simultaneous occupation of the $i$ th and $(i+1)$-th sites arisen from the particle hopping with exclusion. $F_{i, A}$ and $F_{i, D}$, respectively, represent the gain and the loss terms for $\left\langle\tau_{i} \tau_{i+1}\right\rangle$, due to the attachment and the detachment processes. The gain terms, $F_{i, \text { in }}, F_{i, A}$, and loss terms, $F_{i, \text { out }}, F_{i, D}$, are, respectively, given in Tables I and II and Tables III and IV. Column 2 of Tables I and II and Tables III and IV represents the gain and the loss terms as a linear combination of three- and four-point correlators, respectively, whereas column 3 of Tables I and II and Tables III and IV gives the corresponding terms as a function of oneand two-point correlator functions, respectively, obtained by applying Eqs. (30)-(33). Substituting $F_{i, \text { in }}, F_{i, \text { out }}, F_{i, A}$, and $F_{i, D}$ from the third column of Tables I-IV, into Eq. (43), we can obtain the temporal evolution of the two-point correlator function $f_{i}$ as a nonlinear combination of density and correlator function.

We now move to step 2 . To obtain the solution of the average density $\rho$ and the correlator function $f$, the discrete form of the master equations is first converted into their continuum form. For this, we coarse-grain the discrete lattice with lattice constant $\epsilon=1 / N \rightarrow 0$ and rescale the time variable as $t^{\prime}=$ $t / N$. For large systems $N \gg 1$ and $\epsilon \ll 1$, a quasicontinuous variable $x=i \epsilon$ is introduced which denotes the position of the particles on the lattice. To observe the competing interplay between the boundary and bulk dynamics, we also rescale the attachment and detachment rates as in Eq. (23). Taylor's expansion of average two-point correlator function $f(x) \equiv f_{i}$ in the leading powers of $\epsilon$ is given as

$$
f(x \pm n \epsilon)=f(x) \pm n \epsilon \partial_{x} f(x)+\frac{1}{2}(n \epsilon)^{2} \partial_{x}^{2} f(x)+O\left(\epsilon^{3}\right) .
$$

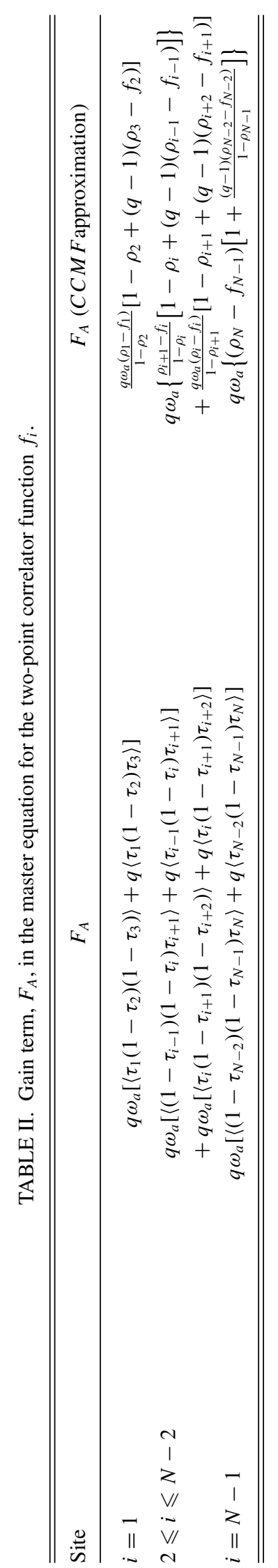


TABLE III. Loss term $F_{\text {out }}$ in the master equation for the two-point correlator function $f_{i}$.

\begin{tabular}{lcr}
\hline \hline Site & $F_{\text {out }}$ & $F_{\text {out }}(C C M F$ approximation $)$ \\
\hline $1 \leqslant i \leqslant N-2$ & $r\left[\left\langle\tau_{i} \tau_{i+1}\left(1-\tau_{i+2}\right)\right\rangle\right]+(1-r)\left\langle\tau_{i} \tau_{i+1}\left(1-\tau_{i+2}\right) \tau_{i+3}\right\rangle$ & $\frac{f_{i}\left(\rho_{i+1}-f_{i+1}\right)}{\rho_{i+1}}\left[r+\frac{(1-r)\left(\rho_{i+3}-f_{i+2}\right)}{\left(1-\rho_{i+2}\right)}\right]$ \\
$i=N-2$ & $r\left\langle\tau_{N-2} \tau_{N-1}\left(1-\tau_{N}\right)\right\rangle$ & $\frac{r f_{N-2}\left(\rho_{N-1}-f_{N-1}\right)}{\rho_{N-1}}$ \\
$i=N-1$ & $r \beta\left\langle\tau_{N-1} \tau_{N}\right\rangle$ & $r \beta f_{N-1}$ \\
\hline \hline
\end{tabular}

Utilizing the above equation and Taylor's series expansion of the average density from Eq. (22) in the correlated cluster mean-field equations for the average bulk density $\rho$ and the bulk correlator function $f$, we obtain the following continuum form in the steady state:

$$
\left[\begin{array}{ll}
g_{1} & g_{2} \\
h_{1} & h_{2}
\end{array}\right]\left[\begin{array}{l}
\frac{\partial \rho}{\partial x} \\
\frac{\partial f}{\partial x}
\end{array}\right]-\frac{\epsilon}{2} \frac{\partial^{2} \rho}{\partial x^{2}}\left[\begin{array}{l}
1 \\
1
\end{array}\right]=\left[\begin{array}{l}
S_{i, A}-S_{i, D}-N g_{0} \\
F_{i, A}-F_{i, D}-N h_{0}
\end{array}\right],
$$

where the functions $g_{k}(\rho, f)$ and $h_{k}(\rho, f)$, for $k=0,1,2$ are given in Table $\mathrm{V}$ and Table VI, respectively. In the thermodynamic limit, the functions $g_{0}, h_{0} \rightarrow 0$ and thus right-hand side of Eq. (45) converge to the pure source and sink terms. The continuum steady-state master equations of $\rho$ and $f$ for the boundary sites are given in Appendix B. The pair of coupled equations, Eqs. (48) and (B2)-(B4), can, respectively, be solved to provide the left and right boundary conditions. Further, we discuss the constant solution independent of the left and the right boundary conditions. The zeros of the source-sink term, $S_{i, A}-S_{i, D}=0$, given by the equation

$$
\begin{aligned}
& \rho+(r-1) f\left[2+\frac{(r-1) f}{\rho}\right] \\
& -K\{1-\rho+2(q-1)(\rho-f) \\
& \left.+(q-1)^{2}\left[\frac{(\rho-f)^{2}}{1-\rho}\right]\right\}=0,
\end{aligned}
$$

yield the Langmuir isotherm solution $\rho=\rho_{l}$, where $f$ is given by Eq. (36). The solution $\rho_{l}$ depends on the interaction energy $E$ and the binding constant $K$.

\section{A. Special cases}

(a) $E=0$ : When there are no interactions, i.e., $E=0$, the steady-state master equation of the density and the correlator function from Eq. (45), respectively, reduce to

$$
\partial_{x} \rho-\partial_{x} f=\frac{\epsilon}{2} \frac{\partial^{2} \rho}{\partial x^{2}}+\Omega_{a}(1-\rho)-\Omega_{d} \rho
$$

and

$$
\begin{aligned}
& \left(\frac{-f^{2}}{\rho^{2}}\right) \frac{\partial \rho(x, t)}{\partial x}+\left(\frac{f}{\rho}+\frac{\rho-f}{1-\rho}\right) \frac{\partial f(x, t)}{\partial x} \\
& =\frac{\epsilon}{2} \frac{\partial^{2} \rho}{\partial x^{2}}+2 \Omega_{a}(\rho-f)-2 \Omega_{d} f-N\left(\rho^{2}-f\right)
\end{aligned}
$$

The left and right boundary conditions for $\rho$ and $f$ given in Appendix B reduce to $\rho(0)=\alpha, \rho(1)=1-\beta, f(0)=$ $\alpha^{2}$, and $f(1)=(1-\beta)^{2}$. In the thermodynamic limit, the coefficient of $N$ in Eq. (48) must approach 0 , thus yielding the relation $f=\rho^{2}$. Utilizing the relation in Eq. (47), we get

$$
\frac{1}{\Omega_{d}}(2 \rho-1) \partial_{x} \rho=K(1-\rho)-\rho,
$$

which is the the corresponding equation of TASEP-LK system [15].

(b) $E=-\infty$ : When the interactions are highly repulsive, i.e., $E \rightarrow-\infty$, the two-point correlator function, $f$, vanishes and the steady-state master equation for the average density obtained from the continuum-correlated cluster mean-field approximation in the limit $\epsilon \rightarrow 0$ becomes

$$
\frac{1}{\Omega_{D}} \partial_{x}\left[\frac{\rho(1-2 \rho)}{1-\rho}\right]=K \frac{(1-2 \rho)^{2}}{(1-\rho)}-\rho,
$$

which matches exactly with the corresponding equation of the noninteracting TASEP-LK system of dimers [41]. The left boundary condition gives $\rho(0)=\alpha /(1+\alpha)$ and the right boundary condition gives $\rho_{N-1}=\beta \rho_{N}$, which again matches with the corresponding equations of noninteracting TASEP of dimers with LK [41]. Unlike the special cases, $E=0$ and $E \rightarrow-\infty$, the coupled system of second-order nonlinear differential equations, Eq. (45), respecting the left and right boundary conditions [obtained from solving Eqs. (48) and (B4)], for a given finite nonzero interaction energy, can not be solved analytically. They are required to be solved numerically for obtaining the density $\rho(x)$ and the two-point correlator $f(x)$. The steady-state solution, $\rho(x)$, is classified as low-density, high-density or a shock, depending on whether the boundary layer confines to the extreme right, to the extreme left, or between positions $0<x<1$ of the lattice length. The steady-state solution will be close to Langmuir

TABLE IV. Loss term, $F_{D}$, in the master equation for the two-point correlator function $f_{i}$.

\begin{tabular}{lcc}
\hline \hline Site & $F_{D}$ & $F_{D}(C C M F$ approximation $)$ \\
\hline$i=1$ & $r \omega_{d}\left(\left\langle\tau_{1} \tau_{2}\right\rangle+(r-1)\left\langle\tau_{1} \tau_{2} \tau_{3}\right\rangle\right)$ & $r \omega_{d} f_{1}\left[1+(r-1) \frac{f_{2}}{\rho_{2}}\right]$ \\
$2 \leqslant i \leqslant N-2$ & $r \omega_{d}\left[2\left\langle\tau_{i} \tau_{i+1}\right\rangle+(r-1)\left(\left\langle\tau_{i-1} \tau_{i} \tau_{i+1}\right\rangle+\left\langle\tau_{i} \tau_{i+1} \tau_{i+2}\right\rangle\right)\right]$ & $r \omega_{d} f_{i}\left[2+(r-1)\left(\frac{f_{i-1}}{\rho_{i}}+\frac{f_{i+1}}{\rho_{i+1}}\right)\right]$ \\
$i=N-1$ & $r \omega_{d}\left[\left\langle\tau_{N-1} \tau_{N}\right\rangle+(r-1)\left\langle\tau_{N-2} \tau_{N-1} \tau_{N}\right\rangle\right]$ & $r \omega_{d} f_{N-1}\left[1+(r-1) \frac{f_{N-2}}{\rho_{N-1}}\right]$ \\
\hline \hline
\end{tabular}


TABLE V. Terms in the continuum limit of the steady-state master equation for $\rho_{i}$ obtained under the CCMF approximation.

\begin{tabular}{lccc}
\hline \hline Site & $g_{0}(\rho, f)$ & $g_{1}(\rho, f)$ & $g_{2}(\rho, f)$ \\
\hline$i=2$ & $\frac{f(\rho-f)}{\rho}\left\{(1-r)-\left[\frac{(2-q-r)(\rho-f)}{1-\rho}\right]\right\}$ & $\left\{\frac{(\rho-f)(2-f-\rho)[f(q+r-2)+\rho(1-q)]}{\rho(1-\rho)^{2}}\right\}-1$ & $\frac{f(f-\rho)[r-1+f(q+r-2)+\rho(3-q-2 r)]}{\rho(1-\rho)}$ \\
$3 \leqslant i \leqslant N-2$ & 0 & $\frac{\partial J}{\partial \rho}$ & $\frac{\partial J}{\partial f}$ \\
$i=N-1$ & $\frac{(f-\rho)^{2}[f(2-q-r)+\rho(q-1)]}{\rho(1-\rho)}$ & $\frac{(2-q-r)(f-\rho)^{2} f}{\rho^{2}(1-\rho)}-\frac{(r-1) f^{2}}{\rho^{2}}-1$ & $\frac{3 f^{2}(q+r-2)-f[2-\rho(13-6 q)+r(7 \rho-2)]}{\rho(1-\rho)}-\frac{\rho[r-2+\rho(7-3 q-3 r)]}{\rho(1-\rho)}$ \\
\hline \hline
\end{tabular}

isotherm density $\rho_{l}$ if the solution satisfies neither the left nor the right boundary conditions.

\section{PHASE DIAGRAMS AND DENSITY PROFILES}

We construct the phase diagrams, the density, the current, and the correlation profiles of the interactive TASEP-LK system for different values of interaction strength and binding constant, using the continuum-correlated cluster mean-field method and further compare them with the corresponding results from the continuum SMF approximation. We also validate the numerical results of the continuum CCMF approach with Monte Carlo simulations for a system size $L=1000$. Similarly to the case of the simple TASEP-LK system, where the system's properties depend significantly on the binding constant $K$, we now explore the steady-state properties by splitting the discussion into two cases, based on the value of the binding constant $K$.

\section{A. $K=1$}

In the absence of interactions, the phase diagram of simple TASEP-LK system for $\Omega_{a}=\Omega_{d}=\Omega$ consists of seven different phases, namely, low-density (LD), high-density (HD), maximal current (MC), and four coexistence phases: LD-HD, LD-MC, LD-MC-HD, and MC-HD [see Fig. 4(a). The consideration of nearest-neighbor particle interactions in the system completely changes the topology of the phase diagram. The inset of the interactions whether repulsive or attractive reduces the number of possible phases from seven to three (see Fig. 4): LD, HD, and a shock (S) phase. In the presence of interactions, the MC region of the simple TASEP-LK system is replaced by either an HD phase, in the case of attractions, or an LD phase, for the case of repulsions. The characteristics of the HD and the LD phases, arrived at in the place of the MC phase, are different from the general HD and LD phases, where the density profiles, respectively, satisfy the right and the left boundary conditions [15]. To distinguish such HD and LD phases from the general HD and LD phases, we term them to be the high-density Meissner $\left(H D_{M}\right)$ and low-density Meissner $\left(L D_{M}\right)$ phase, respectively, due to their similarity with the Meissner phase found in the superconducting materials [15]. The particle density in the $L D_{M}$ and $H D_{M}$ phases is independent of both boundary conditions and is attracted to the Langmuir isotherm density $\rho_{l}$ [see Eq. (47)]. For the case of attractive interactions, we obtain $\rho_{l}>0.5$, and thus the MC phase of the simple TASEP-LK system gets replaced by the $H D_{M}$ phase, whereas for the repulsions, we get $\rho_{l}<0.5$, and thus the $L D_{M}$ phase occupies the place of the MC phase. As a consequence, the $S$ phase for attractive interactions can be any of the two coexistence phases, LD-HD and LD- $H D_{M}$, while, for the repulsive interactions $S$ phase can be either LD-HD or $L D_{M}$-HD. The particle density in the LD and the HD phase is, respectively, given as $\rho<\rho_{l}$ and $\rho>\rho_{l}$. All the phases in interactive systems with $\Omega_{a}=\Omega_{d}$ have the same characteristics as the corresponding phases in the simple TASEP-LK model but with $\Omega_{a} \neq \Omega_{d}[14,15]$. Similarly, the phase boundaries are obtained by matching the corresponding currents as in Ref. [15].

Figures 4(b)-4(d) and 4(e) and 4(f), respectively, show the phase diagrams for different attractive and repulsive interaction strength obtained from the continuum SMF, continuum CCMF, and Monte Carlo simulations (MCSs). The results obtained from the continuum CCMF approximation are in good agreement with the simulation results, whereas the phase boundaries obtained from the continuum SMF approximation mismatch the computer simulations. These deviations become more significant with an increase in the strength of interactions. For instance, in Fig. 4(c) the SMF approximation predicts an extra phase, namely, the LD phase, for smaller values of $\alpha$ and larger values of $\beta$, but the phase does not exist according to the computer simulations and the continuum CCMF theory. To further validate our results of the CCMF theory with the simulations, we plot the density profiles for different attractive and repulsive interaction strength, respectively, in the first row of Figs. 5 and 6. It is clear from the figures that the density profiles predicted from the CCMF approach agree well with the simulations. Moreover, unlike the case of no interactions, the density profiles for interactive systems are not always the linear lines [15]. It is also verified from the density profiles in Figs. 5(c) and 5(d) and 6(b) and 6(d) that, at certain parameters, the SMF and the CCMF approach predict completely different phases. Moreover, in the case when both theories predict the same phase, their corresponding density profiles deviate largely from each other; see Figs. 5(a) and 5(b) and 6(a) and 6(e).

We now discuss how the variation in the interaction energy affects the topology of the phase diagrams. The increment in the attractive interaction strength progressively shrinks the LD region near the $\beta$ axis, and at a critical interaction strength, the LD region collapses leading to a phase diagram with phases $S$, $\mathrm{HD}$, and $H D_{M}$; see Figs. 4(b)-4(d). A further increase in the positive energy $E$ leads to the vanishing of the shock phase at some critical interaction strength, and HD and $H D_{M}$ phases completely dominate the phase diagram. The paramountcy of only HD phases for large attractive interactions is also physically justified because attractive interactions favor the clustering of particles, which leads to a high-density region. Similarly, on decreasing $E$ from 0 , first, the HD phase shrinks, the $S$ phase gets a shift, and the LD region expands. Ultimately, at some critical interaction strength, the HD phase disappears, and the region gets replaced by an $\mathrm{S}$ phase; see 


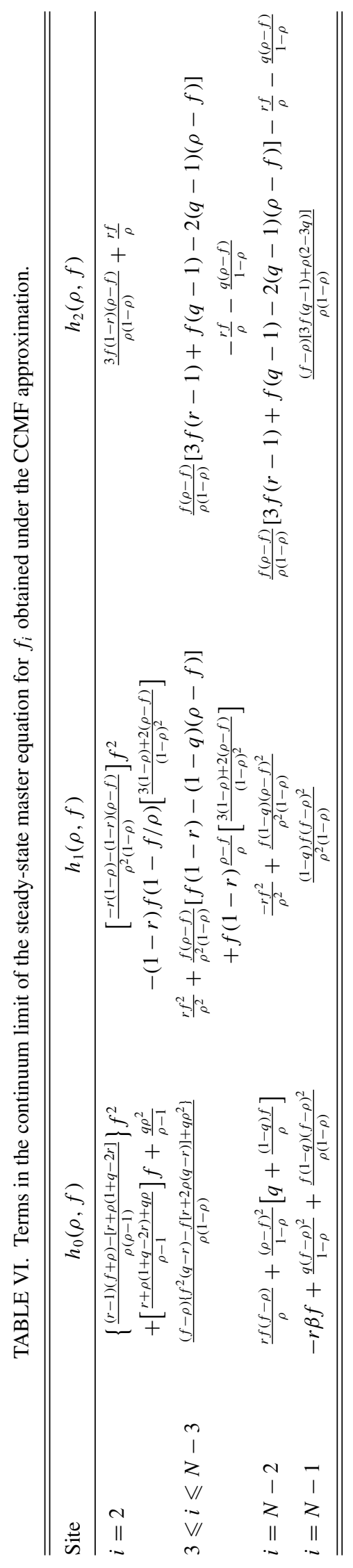

Figs. 4(e) and 4(f). On further decreasing $E$, the $\mathrm{S}$ phase shrinks and finally vanishes at some another critical interaction strength. For $E \ll 0$, only low-density phases prevail in a phase diagram, which is also physically justified because the repulsions cause the particles to fall apart from each other. To further visualize how the interaction energy shifts the phases, we plot the density profiles for fixed left and right reservoir densities but different interaction energy in Figs. 7(a) and 7(b). Clearly, for the attractive interactions, on increasing $E$, an LD profile moves to an HD profile via a shock profile. Similarly, in the case of repulsions, the density profile moves from HD $\rightarrow \mathrm{S} \rightarrow \mathrm{LD}$, with the increase in the repulsive strength.

From a biological point of view, the nonexistence of the MC phase for interactive TASEP-LK system in an ideal situation $K=1$, i.e., symmetric attachment and detachment rates, implies that the motor proteins do not operate at the maximal current conditions. Further, it also sheds information that in traffic flow, the presence of interactions does not favor the maximum flow of vehicles for $K=1$.

\section{B. $K \neq 1$}

We now consider the case when the attachment and detachment rates are asymmetric implying $K \neq 1$. Here the maximum number of possible distinct phases can reach seven, unlike the corresponding case of the simple TASEP-LK system, where only three distinct phases can exist. The topology of the phase diagram depends on the maximum $\left(\rho_{\max }\right)$ of the current-density relation and the zeros $\left(\rho_{l}\right)$ of the source-sink term [41]. In general, $\rho_{l}$ depends on the binding constant $K$ and the interaction energy $E$, while $\rho_{\max }$ is only a function of $E$. Equating $\rho_{\max }$ with $\rho_{l}$ yields a critical binding constant $K^{*}$ depending only on $E$; see Fig. 8 . The results from both the theories match with the MCS for attractive and moderate repulsions, whereas, for the case of strong repulsions, the SMF results start deviating largely from the simulations. We found that for the case of attractive interactions $K^{*}<1$, while for the repulsions $K^{*}>1$, whereas $K^{*}=1$, in the case of no interactions. Interestingly, the maximal-current phase in a phase diagram exists only for $K=K^{*}[14,41]$. The reason for $K^{*}<1$ for attractive interactions can be understood as follows. The attractive interactions lead to the clustering of particles, thus increasing the density in the lattice. For obtaining the maximal current at $K^{*}$, the density should be decreased to $\rho_{\max }=0.5$, which is possible if the attachment rate is decreased or if the critical binding constant becomes less than 1. Similarly, the repulsive interactions favor the low density in the system, so to achieve the maximal current, the attachment rate of particles must be increased, which is possible with the increase in the binding constant $K$. Thus, $K^{*}>1$, for the repulsions. Moreover, at $K=K^{*}$, in total, seven distinct phases, LD, HD, MC, LD-HD, LD-MC, LDMC-HD, and MC-HD, can exist in a phase diagram [see Fig. 9(b)]. When $K<K^{*}$, the isotherm density is less than the maximal density. In this case, the lattice act as a repeller, and the MC phase is replaced by a low-density Meissner $\left(L D_{M}\right)$ phase. In this case, the possible phases, including the Meissner phase, are the LD, $L D_{M}$, HD, and S phase [see Fig. 9(d)]. For $K>K^{*}$, the Langmuir isotherm density becomes larger 
(a)
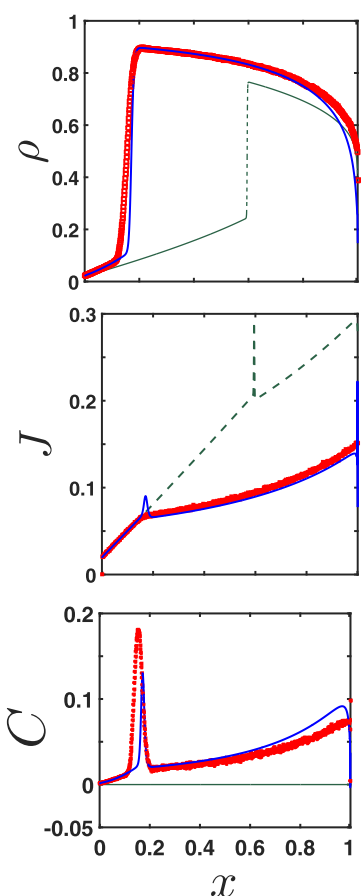

(b)
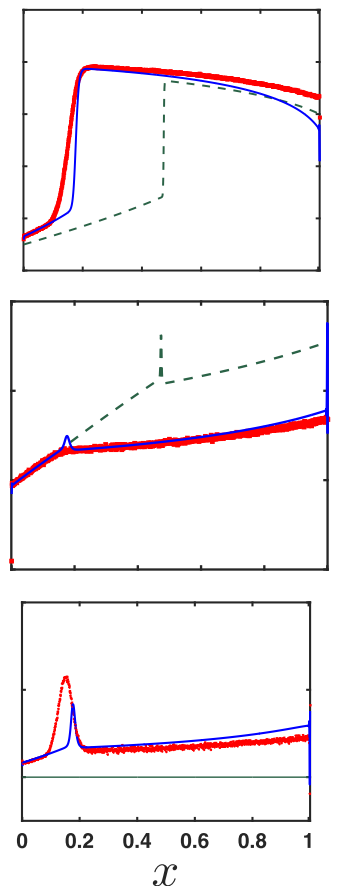

(c)
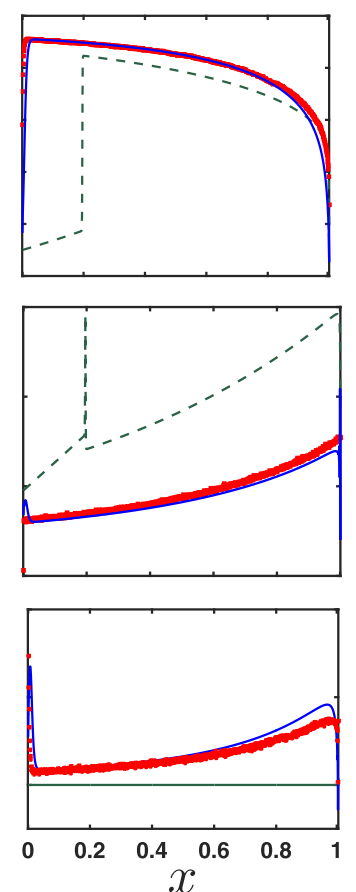

(d)
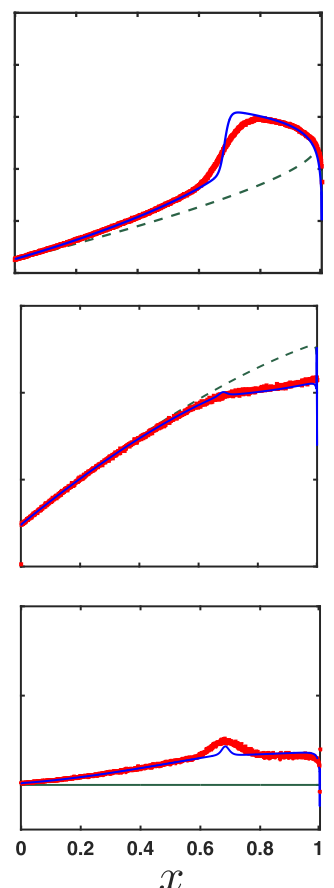

(e)
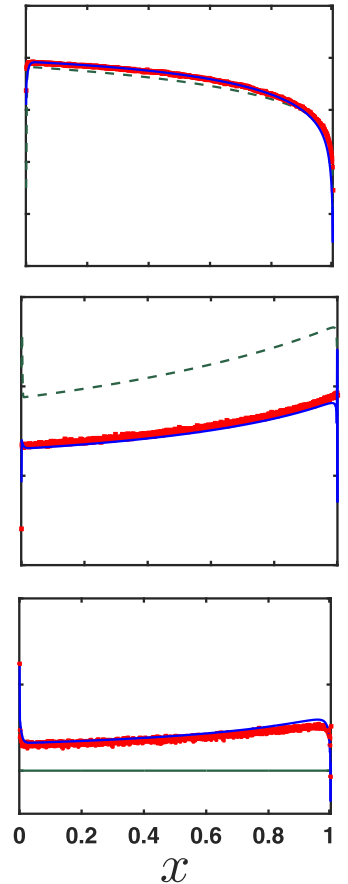

FIG. 5. Average density (first row), current (second row), and the correlation (third row) profiles for $\Omega_{d}=0.3, K=1$ and (a) $E=$ $1.6 k_{B} T, \alpha=0.02, \beta=0.6$; (b) $E=1.0 k_{B} T, \alpha=0.1, \beta=0.4$; (c) $E=1.6 k_{B} T \alpha=0.1, \beta=0.8$; (d) $E=0.6 k_{B} T \alpha=0.05 \beta=0.7$; (e) $E=1 k_{B} T \alpha=0.3 \beta=0.8$. The dashed and solid lines, respectively, denote the continuum SMF and continuum-correlated cluster mean-field results. Symbols are the results of Monte Carlo simulations with a lattice site of 1000 sites, averaged over $5 \times 10^{9}$ simulations.

(a)
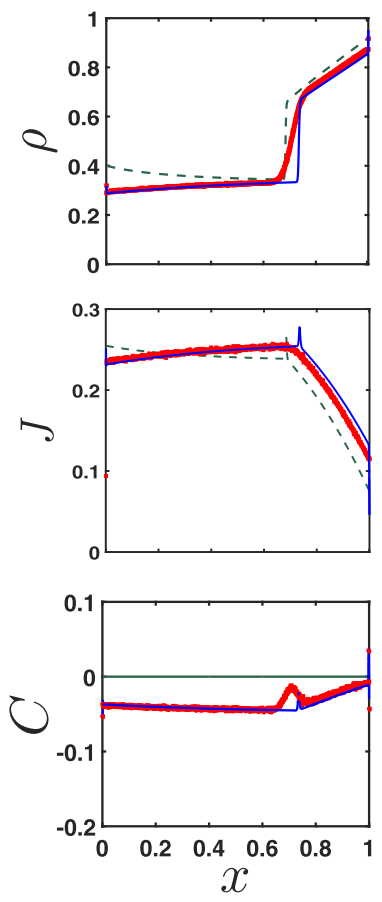

(b)
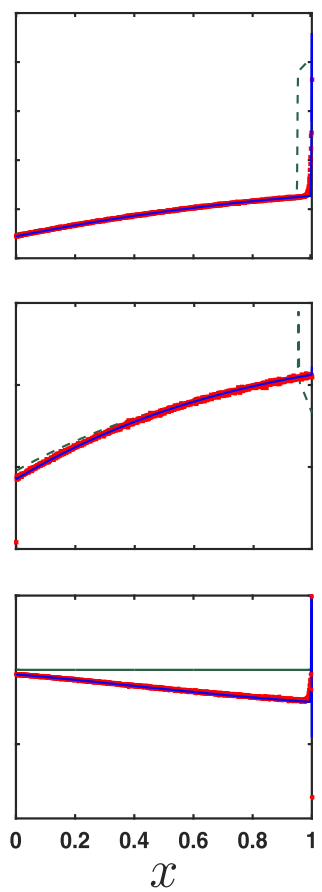

(c)
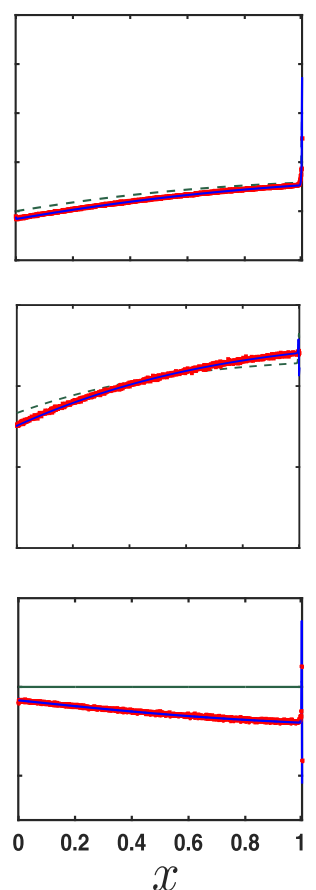

(d)
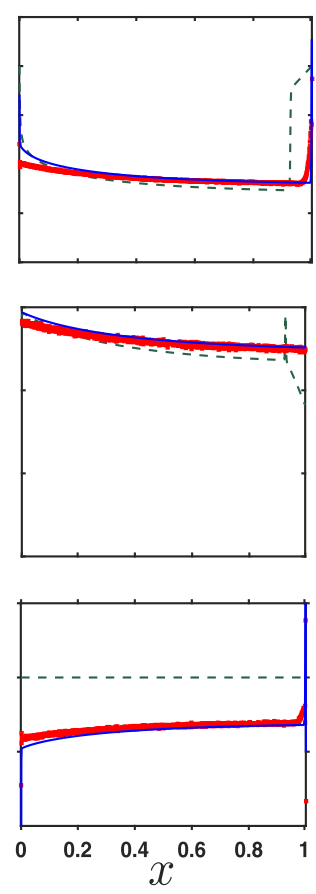

(e)
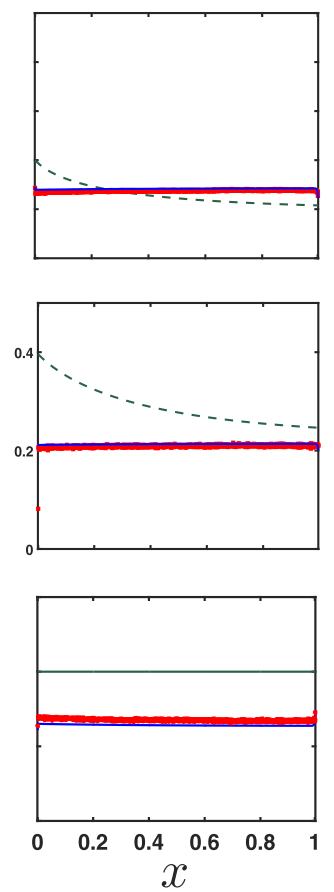

FIG. 6. Average density (first row), current (second row), and the correlation (third row) profiles for $\Omega=0.3, K=1$, and repulsive interaction strength (a) $E=-1.0 k_{B} T, \alpha=0.4, \beta=0.08$; (b) $E=-1.6 k_{B} T, \alpha=0.1, \beta=0.18$; (c) $E=-1.0 k_{B} T, \alpha=0.2, \beta=0.4$; (d) $E=-1.6 k_{B} T \alpha=0.8, \beta=0.2$; (e) $E=-3 k_{B} T, \alpha=0.4, \beta=0.6$. The dashed and solid lines, respectively, denote the continuum SMF and continuum-correlated cluster mean-field results. Symbols are the results of Monte Carlo simulations with a lattice site of 1000 sites, averaged over $5 \times 10^{9}$ simulations. 
(a)

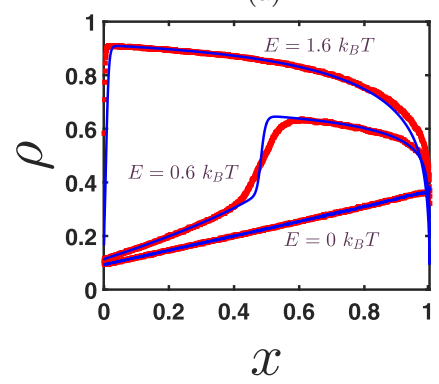

(b)

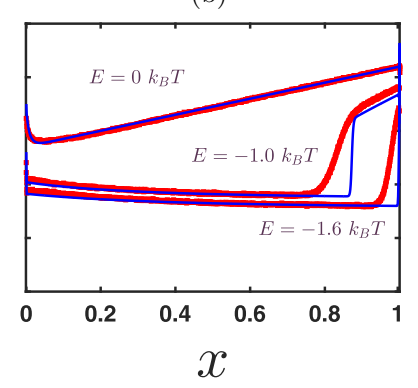

FIG. 7. Density profiles for $K=1, \Omega_{d}=0.3$ and (a) attractive interaction energy for a fixed $\alpha=0.1, \beta=0.7$; (b) repulsive interaction energy for a fixed $\alpha=0.7, \beta=0.16$. Solid lines indicate the correlated cluster mean-field results. Symbols represent the simulation results.

than the maximal density, $\rho_{\max }$. Hence, the phase diagram can consist of the LD, $H D_{M}$, HD, and S phase [see Fig. 9(c)].

The existence of the MC phase in the presence of interactions for the case of asymmetric attachment and detachment rates has physical consequences. In real situations of the biological and vehicular transport processes, particles attach and detach from the track with asymmetric rates. The existing literature on the simple TASEP-LK system informs us that the MC phase cannot exist for $K \neq 1$ [see Fig. 9(a) [14]]. However, in accordance with the experiments, which suggest interactions among particles, our proposed theory predicts that the interactions favor the maximal particle current conditions for asymmetric LK rates.

\section{CURRENT AND CORRELATION}

We now utilize the density and the two-point correlator obtained from the solution of the system of equations [Eq. (45), to calculate the particle current (34)] in the normalized lattice $[0,1]$. The second row of Figs. 5 and 6 , respectively, show the current profiles for different attractive and repulsive interaction strength. It is clear from the figures that the

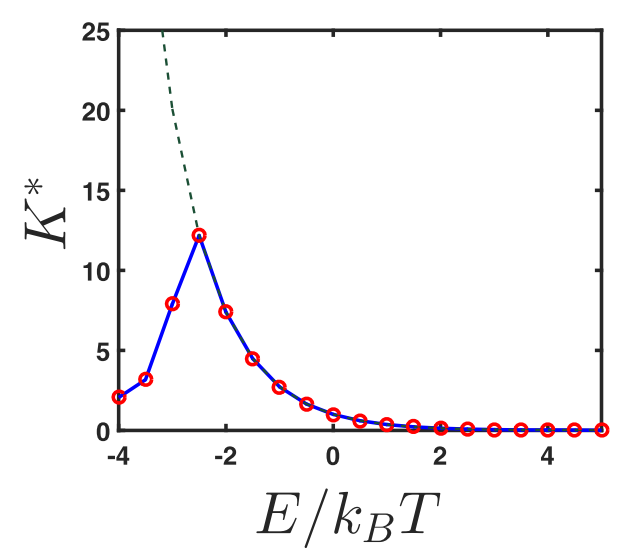

FIG. 8. Critical binding constant $\left(K^{*}\right)$ as a function of interaction energy $E$. Symbols are the results of Monte Carlo simulations with a lattice site of 1000 sites, averaged over $5 \times 10^{9}$ simulations. Dashed and solid lines, respectively, represent the SMF and correlated cluster mean-field results.
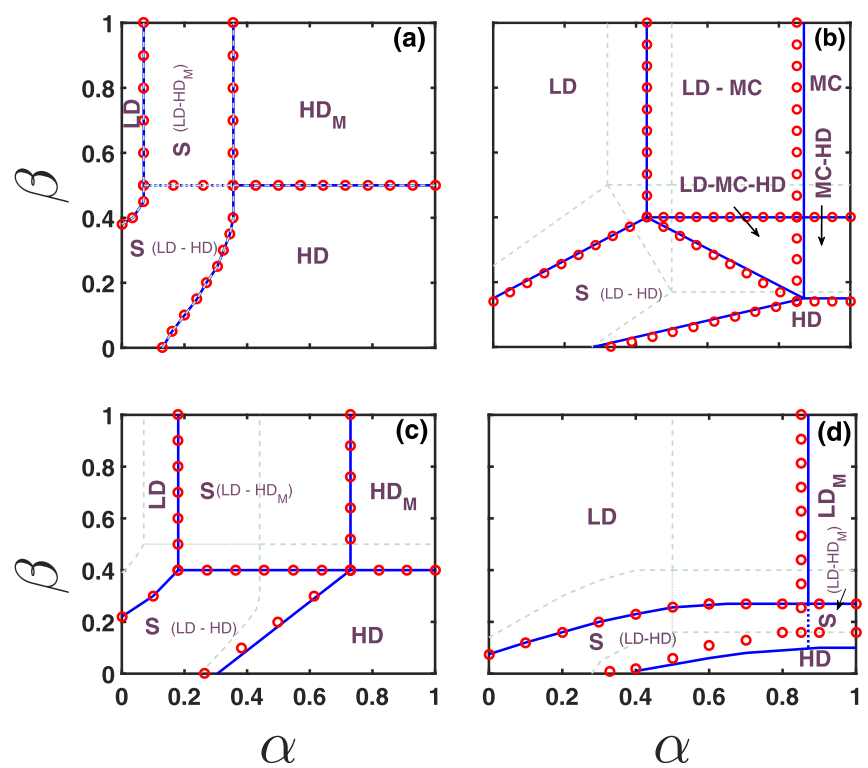

FIG. 9. Phase diagrams for interactive TASEP with mutually interactive Langmuir kinetics for $\Omega_{d}=0.1$ and (a) $E=$ $0.0 k_{B} T, K=2.7182 ; E=-1.0 K_{B} T$ and (b) $K^{*}=2.7182 ;$ (c) $K=4>K^{*}$; (d) $K=1.5<K^{*}$. Solid and dashed lines, respectively, indicate the correlated cluster mean-field and SMF results. Symbols represent the simulation results.

particle current obtained from the continuum CCMF approach matches well with the simulations, while the SMF approach either underestimates or overestimates the particle current. To measure the impact of correlations into the system, we consider a two-point correlation formula

$$
C_{i}=\left\langle\tau_{i} \tau_{i+1}\right\rangle-\rho_{i} \rho_{i+1}=f_{i}-\rho_{i} \rho_{i+1},
$$

where $i=1,2, \ldots, N-1$. Clearly, under the SMF approximation $f_{i}=\rho_{i} \rho_{i+1}$, implying $C=0$, for any value of interaction energy. However, in an interactive system, the correlations are present and thus $f_{i} \neq \rho_{i} \rho_{i+1}$. The CCMF approach takes into account the correlations, and under the approach, we obtain $C \neq 0$ in the presence of interactions (see the third row of Figs. 5 and 6). The sign of the function $C$ depends on the nature of interaction energy $E$. For the case of attractive interactions, where particles tend to bind together, a particle's occupancy at a site depends highly on its neighboring sites, and thus, in this case, one expects $C>0$. However, in the case of repulsions, there is less probability for two particles to be neighbor of each other, and thus the correlation must be negative. The above physical intuition on the sign of function $C$ is also reflected and validated by the correlation profiles obtained numerically from the CCMF approach and the computer simulations. It is clear from the third row of Figs. 5 and 6, respectively, that $C>0$ for $E>0$, while $C<$ 0 for $E<0$. Unlike the bulk conserved interactive TASEP system, the correlation profiles, here, are not always the constant and the straight lines [28,29]. Moreover, we found that the correlation function reaches its maximum value at the domain wall positions. The little discrepancies in the results of our proposed approach and the simulations are due to the consideration of only nearest-neighbor correlations by the 
(a)

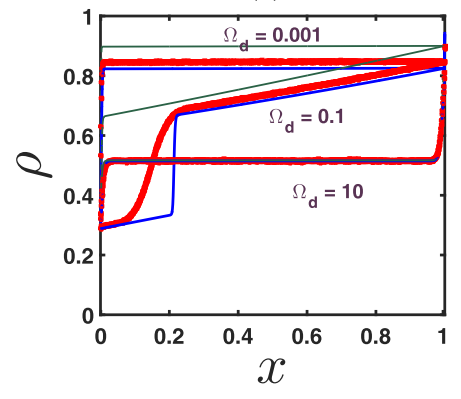

(b)

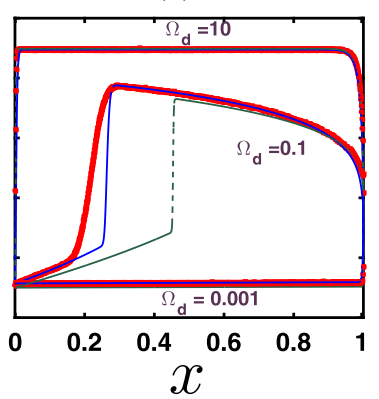

FIG. 10. Average density profiles computed by MCS (symbols), SMF (dashed lines), and correlated cluster mean-field (solid lines) for $K=3$ and for different detachment rates with (a) $E=$ $0.6 k_{B} T, \alpha=0.1$, and $\beta=0.6$ (b) $E=-1.0 K_{B} T, \alpha=0.4$, and $\beta=0.1$.

approach and the finite-size effect, which we discuss in the next section.

\section{EFFECT OF THE LK RATES AND THE FINITE SYSTEM SIZE}

We now discuss the effect of varying the LK rates on the phase boundaries of the interactive system. We exemplify the results by fixing the binding constant $K=3$. Figures 10 (a) and 10(b) show the density profiles for different values of kinetic rates and interaction strength. In the case of attractive interactions, on increasing $\Omega_{d}$, an LD region first shifts to an $\mathrm{S}$ phase, and on further increase in $\Omega_{d}$, the phase changes to an HD region; see Fig. 10(a). Similarly, for the case of repulsive interactions, increasing $\Omega_{d}$ from 0 to larger values shifts the density profile from HD $\rightarrow \mathrm{S} \rightarrow$ LD; see Fig. 10(b). In the figure, the results from the CCMF approximation are in good agreement with MCS even for all ranges of kinetic rates. When the kinetic rates approach 0 , the density profiles match with the corresponding profiles of interactive TASEP system; while for large kinetic rates, the bulk density approaches to the equilibrium density of the stochastic attachment-detachment process. Further, by comparing the results of Fig. 10 with Fig. 7, it is concluded that the effect of increasing $\Omega_{d}$ with a fixed energy $E$ has the same consequences on the density

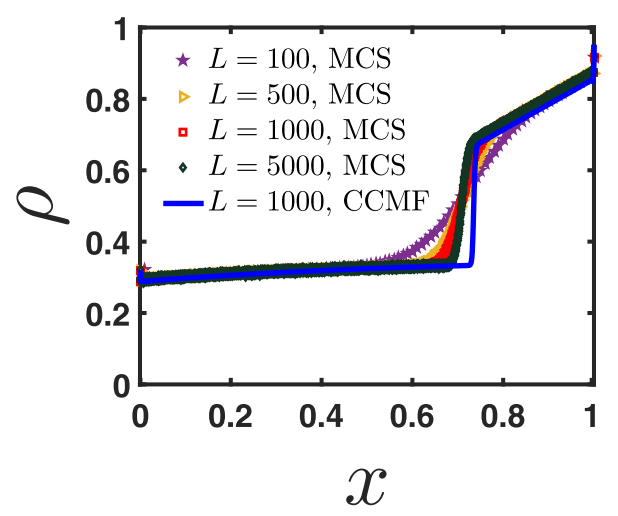

FIG. 11. Effect of system size on the shock profile for $E=$ $-1.0 k_{B} T, \alpha=0.4, \beta=0.08, K=1, \Omega_{d}=0.3$.

profiles as with increasing $E$ from 0 or decreasing $E$ from 0 , with a fixed $\Omega_{d}$.

We also observe the effect of the system size on the position of the domain wall as obtained from the Monte Carlo simulations. It is clear from Fig. 11 that the sharpness in the steep rise of the shock increases as one increases the number of lattice sites, and it also justifies that our chosen system size $L=1000$ is appropriate enough to study the interactive TASEP-LK system.

\section{SUMMARY AND DISCUSSIONS}

To summarize, we have considered the nearest-neighbor interactions in a generic model for nonconserving driven diffusive systems. The interactions are encountered in a thermodynamically consistent way that affects the dynamical rules as well as hopping rates of both the TASEP and the LK process of the simple TASEP-LK system. Our model is more general and realist8ic than the existing models for interactions in the TASEP-LK system, which considered the nearest-neighbor interactions either only in the TASEP or only in the LK process. We have first analyzed the system with SMF approximation and have shown that its estimation for the maximal particle current, phase diagrams, density, and current profiles deviate largely from the simulations. To overcome the drawbacks of SMF, we proposed an approach, which we call the correlated cluster mean-field theory (CCMF), that exactly considers the correlations between clusters of size two and approximates the correlation between clusters of size greater than $t w o$. Under the approximation, the particle current can be written as a function of density and a two-point correlator function defining the probability of two consecutive occupied sites. In the continuum limit, the master equations for the density and the two-point correlator reduce to a coupled nonlinear second-order partial differential equation with time and space as the continuous variable. We have computed the steady-state properties such as phase diagrams, density, current, and correlation profiles for different interaction strength, kinetic rates, and binding constant $K$ using the CCMF approach and found them to be in very well agreement with the Monte Carlo simulations. Correlations are found to be negative for repulsive interactions, while they are always positive for attractive interactions. Moreover, in the vicinity of shock positions, the correlations are strongest. We have also analyzed the effect of different kinetic rates on the phase boundaries using the CCMF approach and the Monte Carlo simulations. As expected, for $\Omega \rightarrow 0$, the results reduce to the interactive TASEP system without LK, and similarly for larger $\Omega$, the particle density approaches the Langmuir isotherm density. We have also examined the effect of the system size on the density profiles. The results of the CCMF approximation agree well with the Monte Carlo simulations.

Interestingly, we found that unlike the simple TASEP-LK model, in the case of symmetric attachment and detachment rates, the maximal current (MC) phase does not exist in the presence of interactions. However, for the realistic case when the attachment and detachment rates for particles are asymmetric, the MC phase, which did not exist for the simple TASEP-LK system, starts occurring as soon as the interactions are encountered. Our results suggest that the motor proteins 
can experience the maximal current only when the interactions are taken into account, which is an important result from the biological point of view.

\section{APPENDIX A: PARTICLE CURRENT AND ATTACHMENT-DETACHMENT TERMS}

Substituting $\tilde{\tau}_{i}=1-\tau_{i}$ in Eqs. (3)-(9), the expressions for particle current and attachment-detachment terms are simplified to following equations as a linear combination of one-, two-, three-, and four-point correlators:

$$
\begin{aligned}
& J_{i, i+1}=\left\langle\tau_{i}\right\rangle-\left\langle\tau_{i} \tau_{i+1}\right\rangle+(q-1)\left\langle\tau_{i}\left(1-\tau_{i+1}\right) \tau_{i+2}\right\rangle \\
&+(r-1)\left(\left\langle\tau_{i-1} \tau_{i}\right\rangle-\left\langle\tau_{i-1} \tau_{i} \tau_{i+1}\right\rangle\right) \\
&+(2-q-r)\left\langle\tau_{i-1} \tau_{i}\left(1-\tau_{i+1}\right) \tau_{i+2}\right\rangle, \\
& J_{\text {entr }}= \alpha\left\langle\left(1-\tau_{1}\right)\left(1-\tau_{2}\right)\right\rangle+q \alpha\left\langle\left(1-\tau_{1}\right) \tau_{2}\right\rangle \\
&= \alpha\left[1-\left\langle\tau_{1}\right\rangle-(1-q)\left(\left\langle\tau_{2}\right\rangle-\left\langle\tau_{1} \tau_{2}\right\rangle\right)\right], \\
& J_{1,2}=\left\langle\tau_{1}\left(1-\tau_{2}\right)\left(1-\tau_{3}\right)\right\rangle+q\left\langle\tau_{1}\left(1-\tau_{2}\right) \tau_{3}\right\rangle \\
&=\left\langle\tau_{1}\right\rangle-\left\langle\tau_{1} \tau_{2}\right\rangle+(q-1)\left\langle\tau_{1}\left(1-\tau_{2}\right) \tau_{3}\right\rangle, \\
& J_{N-1, N}=\left\langle\left(1-\tau_{N-2}\right) \tau_{N-1}\left(1-\tau_{N}\right)\right\rangle \\
& \quad+r\left\langle\tau_{N-2} \tau_{N-1}\left(1-\tau_{N}\right)\right\rangle \\
&=\left\langle\tau_{N-1}\right\rangle-\left\langle\tau_{N-1} \tau_{N}\right\rangle-(1-r)\left\langle\tau_{N-2} \tau_{N-1}\right\rangle \\
& \quad+(1-r)\left\langle\tau_{N-2} \tau_{N-1} \tau_{N}\right\rangle, \\
& J_{\text {exit }}=\beta\left\langle\left(1-\tau_{N-1}\right) \tau_{N}\right\rangle+r \beta\left\langle\tau_{N-1} \tau_{N}\right\rangle \\
& \quad=\beta\left[\left\langle\tau_{N}\right\rangle+(r-1)\left\langle\tau_{N-1} \tau_{N}\right\rangle\right],
\end{aligned}
$$

[1] S. Katz, J. L. Lebowitz, and H. Spohn, Phys. Rev. B 28, 1655 (1983).

[2] J. Krug, Phys. Rev. Lett. 67, 1882 (1991).

[3] D. Chowdhury, A. Schadschneider, and K. Nishinari, Phys. Life Rev. 2, 318 (2005).

[4] R. A. Blythe and M. R. Evans, J. Phys. A 40, R333 (2007).

[5] B. Schmittmann and R. K. Zia, Phase Transitions Crit. Phenom. 17, 3 (1995).

[6] C. Appert-Rolland, M. Ebbinghaus, and L. Santen, Phys. Rep. 593, 1 (2015).

[7] T. Chou, K. Mallick, and R. Zia, Rep. Prog. Phys. 74, 116601 (2011).

[8] T. Chou and G. Lakatos, Phys. Rev. Lett. 93, 198101 (2004).

[9] S. Klumpp and R. Lipowsky, J. Stat. Phys. 113, 233 (2003).

[10] H. Hilhorst and C. Appert-Rolland, J. Stat. Mech.: Theory Exp. (2012) P06009.

[11] R. Lipowsky, S. Klumpp, and T. M. Nieuwenhuizen, Phys. Rev. Lett. 87, 108101 (2001).

[12] C. T. MacDonald, J. H. Gibbs, and A. C. Pipkin, Biopolymers 6, 1 (1968).

[13] G. Schütz and E. Domany, J. Stat. Phys. 72, 277 (1993).

[14] A. Parmeggiani, T. Franosch, and E. Frey, Phys. Rev. Lett. 90, 086601 (2003).

$$
\begin{aligned}
S_{i, A}= & \omega_{a}\left[1-\left\langle\tau_{i}\right\rangle+(q-1)\left(\left\langle\tau_{i-1}\right\rangle+\left\langle\tau_{i+1}\right\rangle-\left\langle\tau_{i-1} \tau_{i}\right\rangle\right.\right. \\
& \left.\left.\left.-\left\langle\tau_{i} \tau_{i+1}\right\rangle\right)+(q-1)\left\langle\tau_{i-1}\left(1-\tau_{i}\right) \tau_{i+1}\right\rangle\right)\right],
\end{aligned}
$$

and

$$
\begin{aligned}
S_{i, D}= & \omega_{d}\left[\left\langle\tau_{i}\right\rangle+(r-1)\left(\left\langle\tau_{i-1} \tau_{i}\right\rangle+\left\langle\tau_{i} \tau_{i+1}\right\rangle\right.\right. \\
& \left.\left.+(r-1)\left\langle\tau_{i-1} \tau_{i} \tau_{i+1}\right\rangle\right)\right] .
\end{aligned}
$$

\section{APPENDIX B: CONTINUUM-CORRELATED CLUSTER MEAN-FIELD APPROXIMATION OF $\rho(x)$ AND $f(x)$}

The left boundary conditions, $\rho_{1}$ and $f_{1}$, for the system of equations in Eq. (45) can be obtained by simultaneously solving the steady-state master equation for the average density $\rho_{1}$ and average two-point correlator $f_{1}$ :

$$
\begin{gathered}
\left\{\left[1+(q-2) \rho_{1}+(1-q) f_{1}\right]\right\}\left[\alpha+\frac{\left(f_{1}-\rho_{1}\right)}{\left(1-\rho_{1}\right)}\right]=0, \quad(\mathrm{~B} 1) \\
q \alpha\left(\rho_{1}-f_{1}\right)-r f_{1}\left(1-\frac{f_{1}}{\rho_{1}}\right)-(1-r)\left[\frac{f_{1}\left(\rho_{1}-f_{1}\right)^{2}}{\rho_{1}\left(1-\rho_{1}\right)}\right]=0 .
\end{gathered}
$$

Similarly, the right boundary conditions, $\rho_{N}$ and $f_{N}$, for the system of equations in Eq. (45) can be acquired from the simultaneous solution of the steady-state master equation for the average density $\rho_{N}$ and average two-point correlator $f_{N}=$ $f_{N-1}$, respectively, given as

$$
\begin{gathered}
\rho_{N}(1-\beta)+f_{N}[\beta(1-r)+(r-2)]+(1-r) \frac{f_{N}^{2}}{\rho_{N}}=0 \\
{\left[q \rho_{N}+(1-q) f_{N}\right]\left[\frac{\left(\rho_{N}-f_{N}\right)^{2}}{\rho_{N}\left(1-\rho_{N}\right)}\right]-r \beta f_{N}=0}
\end{gathered}
$$

[15] A. Parmeggiani, T. Franosch, and E. Frey, Phys. Rev. E 70, 046101 (2004).

[16] T. Antal and G. M. Schütz, Phys. Rev. E 62, 83 (2000).

[17] V. Popkov, A. Rákos, R. D. Willmann, A. B. Kolomeisky, and G. M. Schütz, Phys. Rev. E 67, 066117 (2003).

[18] A. Seitz and T. Surrey, EMBO J. 25, 267 (2006).

[19] S. Katz, J. L. Lebowitz, and H. Spohn, J. Stat. Phys. 34, 497 (1984).

[20] J. S. Hager, J. Krug, V. Popkov, and G. M. Schütz, Phys. Rev. E 63, 056110 (2001).

[21] S. Klumpp and R. Lipowsky, Europhys. Lett. 66, 90 (2004).

[22] M. Dierl, P. Maass, and M. Einax, Europhys. Lett. 93, 50003 (2011).

[23] M. Dierl, P. Maass, and M. Einax, Phys. Rev. Lett. 108, 060603 (2012).

[24] M. Dierl, M. Einax, and P. Maass, Phys. Rev. E 87, 062126 (2013).

[25] I. Pinkoviezky and N. S. Gov, New J. Phys. 15, 025009 (2013).

[26] Q.-Y. Hao, Z. Chen, X.-Y. Sun, B.-B. Liu, and C.-Y. Wu, Phys. Rev. E 94, 022113 (2016).

[27] H. Teimouri, A. B. Kolomeisky, and K. Mehrabiani, J. Phys. A 48, 065001 (2015). 
[28] D. Celis-Garza, H. Teimouri, and A. B. Kolomeisky, J. Stat. Mech.: Theory Exp. (2015) P04013.

[29] T. Midha, A. B. Kolomeisky, and A. K. Gupta, J. Stat. Mech.: Theory Exp. (2018) 043205.

[30] T. Midha and A. K. Gupta, J. Stat. Phys. 169, 824 (2017).

[31] T. Midha, A. K. Gupta, and A. B. Kolomeisky, J. Stat. Mech.: Theory Exp. 2017, 073202 (2017).

[32] S. Chandel, A. Chaudhuri, and S. Muhuri, Europhys. Lett. 110, 18002 (2015).

[33] A. Vilfan, E. Frey, F. Schwabl, M. Thormählen, Y.-H. Song, and E. Mandelkow, J. Mol. Biol. 312, 1011 (2001).

[34] H. D. Vuijk, R. Rens, M. Vahabi, F. C. MacKintosh, and A. Sharma, Phys. Rev. E 91, 032143 (2015).
[35] A. K. Gupta, J. Stat. Phys. 162, 1571 (2016).

[36] M. Schreckenberg, A. Schadschneider, K. Nagel, and N. Ito, Phys. Rev. E 51, 2939 (1995).

[37] A. Schadschneider, D. Chowdhury, and K. Nishinari, Stochastic Transport in Complex Systems: From Molecules to Vehicles (Elsevier, Amsterdam, 2010).

[38] H. A. Gutowitz, J. D. Victor, and B. W. Knight, Physica D 28, 18 (1987).

[39] I. R. Graf and E. Frey, Phys. Rev. Lett. 118, 128101 (2017).

[40] G. Lakatos and T. Chou, J. Phys. A 36, 2027 (2003).

[41] P. Pierobon, E. Frey, and T. Franosch, Phys. Rev. E 74, 031920 (2006). 